\title{
Morfología larval, clave para inmaduros y ciclos de vida de Dynastes hercules L (D. h. lichyi Lachaume, D. h. occidentalis Lachaume) y de Dynastes neptunus Quenzel (Coleoptera: Melolonthidae: Dynastini) en Colombia
}

\author{
Larval morphology, key to immature and life cycles \\ of Dynastes hercules L (D. h. lichyi Lachaume, D. $h$. \\ occidentalis Lachaume), and of Dynastes neptunus Quenzel \\ (Coleoptera: Melolonthidae: Dynastini) in Colombia
}

\author{
Luis Carlos Pardo-Locarno ${ }^{1}$ \\ Libardo Suárez Fonseca \\ Andrés Felipe Serna \\ 'Doctor en Ciencias, Programa de Agronomía, \\ Universidad del Pacifico, Colombia. \\ E.mail:lcpardo@unpacifico.edu.co \\ ${ }^{2}$ Biólogo, Parques Nacionales Naturales de Colombia, \\ Ministerio del Medio Ambiente, Colombia. \\ E-mail: libardo.suarez@parquesnacionales.gov.co \\ 3 Docente, Centro de Evangelización Fuan \\ XxIII, Palmira, Colombia.
}

\section{Resumen}

Los escarabajos gigantes de la tribu Dynastini reúnen en Colombia tres géneros y 14 especies, de las cuales dos pertenecen al género Dynastes Kirby, 1825: D. hercules (Linné, 1758), que incluye tres subespecies y D. neptunus Quenzel, 1817, todas ellas representativas de la biodiversidad nacional. Sin embargo, hace falta mayor investigación en cuanto a los aspectos biológicos y ecológicos, razón por la cual, esta investigación se propuso desarrollar el ciclo de vida y describir la morfología

Historia del artículo

Fecha de recepción:

28-08-2021

Fecha de aceptación:

11-10-2021

DOI: https://doi.

org/10.47374/

novcol.2021.v16.2006 
larval de Dynastes hercules L. (D. h. lichyi Lachaume y $D$. h. occidentalis Lachaume) y de Dynastes neptunus Quenzel (Coleoptera: Melolonthidae: Dynastini), incluyendo un clave para larvas y pupas y discusión de los caracteres estudiados. La investigación se desarrolló en un laboratorio de bioensayos en Palmira (Valle del Cauca, Colombia. Para cada caso se utilizaron recipientes con sustrato orgánico esterilizado, larvas individualizadas, toma de datos de anchura cefálica, anchura corporal y longitud del cuerpo, cada quince días; las muestras fueron fijadas en solución de formol y se etiquetaron cuidadosamente. El ciclo de vida en los tres casos fue largo, alcanzando una media de 1.5-1.8 años, con una porción de casos que superó los dos años; en cuanto a la morfología larval el número de setas dorso epicraneales y la quetotaxia de los apéndices (tarsúngulo), de los teges, y de la acanthoparia se consideran, por ahora, focales en este género. La morfología larval expuso una gran similitud y afinidad entre las especies estudiadas, descartando por ahora erigir especies entre las subespecies de $D$. hercules. La morfología pupal fue bastante similar, evidenciándose solo sutiles diferencias en dos aspectos de la dorsa de los segmentos abdominales VII y VIII. Tanto en la morfología larval como en la de pupa se evidenció una mayor divergencia en Dynastes (Theogenes) neptunus Quenzel, algo que daría validez a este subgénero tan poco estudiado por los autores. Es recomendable ahondar en aspectos del ciclo biológico de D. neptunus dada la mayor duración del mismo, quizás afectado por la alta temperatura.

Palabras clave: Ciclos de vida, morfología larval, escarabajos gigantes, selvas húmedas.

\section{Abstract}

In Colombia, the giant beetles of the Dynastini tribe are represented by three genera and 14 species. Two of which belong to the genus Dynastes Kirby, 1825: D. neptunus Quenzel, 1817; and D. hercules (Linné, 1758) which also includes three subspecies, all of them, prominent members of national biodiversity. However, there is little knowledge regarding its biological and ecological aspects, which is why this research sets out to examine life cycle and describe larval morphology of following specie: Dynastes hercules (D. h. lichyi Lachaume; and D. h. occidentalis Lachaume) and Dynastes neptunus Quenzel (Coleoptera: Melolonthidae: Dynastini). Research also included a key identification for larvae and pupae and analysis of studied characters. The research was developed in a bioassay laboratory in Palmira (Valle del Cauca Colombia). Using containers as cases for cribs, sterilized organic substrate was set for each individualized larva. Data collection consisted of: cephalic, body width and body length measurements every fifteen days. Afterward, some samples were fixed in formaldehyde solution and carefully labeled. In most cases, life cycle reached an average of 1.5-1.8 years, with a portion of cases exceeding two years. Regarding the larval morphology, the number of dorsal epicranial setae and the ketotaxia of the appendages (tarsungulum); the teges and the acanthoparia are considered, for now, focal in this genus. Larval morphology showed great similarity and affinity between the studied species, ruling out for now to separate species among the subspecies of 
D. Hercules. Pupal morphology was quite similar, showing only subtle differences in two aspects of the back of abdominal segments VII and VIII. In both larval and pupal morphology, a greater divergence was evidenced in Dynastes (Theogenes) neptunus Quenzel, element that could give validity to this subgenus under-estimated by the authors. A deeper study is suggested aiming towards biological aspects of D. neptunus given its longer life cycle, perhaps affected by high temperature.

Keywords: Life cycles, larval morphology, giant beetles, rainforests.

\section{Introducción}

Los escarabajos gigantes de la tribu Dynastini reúnen en Colombia tres géneros y 14 especies (Hno Apolinar-Maria, 1944,1945; Blackwelder, 1944; Figueroa, 1977; Gasca et al., 2018; Huang, 2017; Pardo-Locarno, 1996; 2005; Pardo-Locarno et al., 2020; Restrepo et al., 2003); particularmente el género Dynastes Kirby, 1825 incluye las especies $D$. hercules (Linné, 1758) y D. neptunus Quenzel, 1817 (Blackwelder, 1944; Endrödi, 1985; Pardo-Locarno et al., 2020; Restrepo et al., 2003), consideradas reliquias evolutivas y especies emblemáticas, por su gigantismo, marcado dimorfismo sexual y atractivas facies, que incluyen la mayor cornamenta torácica y cefálica del mundo insectil (María, Hno Apolinar, 1944 -1945; Arrow, 1951, p 101; Eberhard, 1977 - 1980; Emlen et al., 2005).

De estas dos especies, $D$. hercules es la que mayor porte alcanza, la más ampliamente distribuida y diversificada en Centro y
Suramérica, con al menos tres subespecies registradas en Colombia (Chalumeau \& Reid, 2002; Lachaume, 1985; Morón, 2009; Pardo-Locarno et al., 2020); mientras que $D$. neptunus destaca mayormente en los altos andes colombianos, en selvas de niebla por encima de los 1500 msnm (Lachaume, 1985; Pardo-Locarno et al., 2020); no obstante algunos logros nacionales (BallesterosMeza \& Amat-García, 2006; Gasca-Álvarez, 2011; Pardo-Locarno, 2006), la biología y ecología de estos gigantes insectiles sigue siendo tema precario, existiendo además, incertidumbre en torno a la conservación de estos distinguidos miembros del patrimonio biológico nacional (Pardo-Locarno et al., 2020). Ante esta situación, la presente investigación se propuso desarrollar el ciclo de vida y describir la morfología larval de Dynastes hercules L. (las subespecies D. h. lichyi Lachaume y D. h. occidentalis Lachaume) y de Dynastes neptunus Quenzel (Coleoptera: Melolonthidae: Dynastini), incluyendo la elaboración de una clave para larvas y pupas y comentarios sobre los caracteres estudiados.

\section{Materiales y métodos}

La revisión de literatura sobre la biología y ensayos de cría fue lo más amplia posible, enfatizando la revisión de documentos formales con revisión por pares, excluyendo páginas web enfocadas a procesos lúdicos de cría o venta de especímenes.

Aspectos biológicos: comprenden el ciclo de vida y la descripción de larvas. 
Ciclos de vida: el desarrollo de ciclos de vida de los escarabajos gigantes Dynastini es tema complejo y muy extenso (IannaconeOlivier y Soras-Vega, 2010; Morón, 1987; Richter, 1940, 1944, 1966; Snodgrass, $1967 \mathrm{p}$. 226-271), pues se trata de años de cría para lograr adultos, razón por la cual se requirió del apoyo consecutivo de diversos proyectos e instituciones (Pardo-Locarno, 2005). Este aparte incluyó la colecta de parentales, aislamiento en bioterio, obtención de huevos, desarrollo de inmaduros (larva, prepupa, pupa) y emergencia de adultos, todo ello basado en ensayos propios y soportes bibliográficos (Morón y PardoLocarno, 1994; Pardo-Locarno, 1997; 2006). Los parentales de $D$. h. lichyi Lachaume y de D. neptunus se colectaron en el municipio de La Paz (Santander - Colombia) a través de trampeo en luces públicas (abril de 2002) y colectas en la Belleza (Santander - Colombia, diciembre de 2003). Los adultos fueron confinados en recipientes adecuados, dotados de alimento como caña picada y fruta madura. Esta fase contó con el apoyo inter institucional del Instituto de Investigaciones Ambientales del Pacífico (IIAP) y la Corporación Autónoma Regional de la Frontera Nororiental (Corponor). Los parentales de $D$. h. occidentalis Lachaume fueron colectados en La Quebrada Triana, Bajo Dagua, (Valle del Cauca - Colombia) en junio 26 de 2011, con trampas de luz tipo piracicaba (Convenio CVC-AAFP, 2011).

La cría dispuso de un laboratorio de bioensayos particular (espacio de $24 \mathrm{~m}^{2} \mathrm{en}$ la zona rural de Palmira, ciudad ubicada a $985 \mathrm{msnm}$, temperatura media de $24^{\circ} \mathrm{C}$, humedad relativa de $70-80 \%)$. El cuarto de cría estuvo dotado del mobiliario y condiciones de cría propuestos por estudios previos (Morón \& Pardo-Locarno, 1994; Pardo-Locarno, 1997; 2006). En 1993 y 2003, se logró compartir con Miguel Ángel Morón, en Xalapa (Veracruz - México), información sobre toma de datos de crecimiento, manejo del sustrato y gajes de cría, en parte inéditos, a propósito de los estudios de Golofa (Morón \& Pardo-Locarno, 1994) y de D. hyllus Chev (Morón, 1987).

Para procurar la cópula y obtención de huevos, se dispuso de bioterios para adultos, en los cuales se dispuso de alimento (fruta madura) y un sustrato orgánico, conformado por troncos podridos desmenuzados y tratamiento térmico (Morón, 1987; Morón \& PardoLocarno, 1994; Pardo-Locarno, 1997, 2006); se tuvo cuidado de proveer humedad, alimento fresco y retirar adultos débiles o enfermos; se realizó la toma de datos de elongación de corion, emergencia de larvas, de crecimiento en bioterios etiquetados, monitoreadas cada 15 días (datos de anchura cefálica, anchura corporal y longitud corporal). A partir de los datos consignados surgió material para fijado y estudio científico, entre ellos huevos, larvas y pupas.

Morfología larval: las larvas se fijaron así: a) insensibilización por frío, b) tratamiento térmico con agua hervida, c) fijación en solución de formol, alcohol y agua destilada (20-20-60 en volumen), d) almacenamiento en frasco de vidrio con rótulo de localidad, fechas de desarrollo y de fijación (Morón \& Pardo-Locarno, 1994; Pardo-Locarno, 1997; 
2006). En algunos casos se aplicó insuflado parcial (Viñez \& Alvarez, 1972), extrayendo los órganos internos principales (corazón, sistema digestivo, tejido graso, etc.), sin aplicar aire. Algunas pupas y exuvias pupales obtenidas se manejaron de la misma manera. Los adultos se colocaron en la misma solución sin tratamiento térmico; parte de las muestras se depositaron en la Colección Familia Pardo-Locarno (CFPLCOL). Las fases descriptivas de inmaduros se basaron en Costa et al (1988), Morón (1987), Morón \& Pardo-Locarno (1994) y Ritcher $(1940 ; 1944,1966)$. Se realizaron tomas fotográficas de órganos diagnósticos, las estructuras disectadas se depositaron en microviales, con solución de formol. Esta actividad se realizó en varios estereoscopios Nikon $^{\mathrm{R}}$, Olympus ${ }^{\mathrm{R}}$ y Vision Scientific VZE$\mathrm{VZF}^{\mathrm{R}}$ dotados de cámaras digitales.

Marco institucional: la iniciativa de desarrollar protocolos y prospectar la cría de escarabajos gigantes de Colombia, inició entre 1991 y 1992 con el estudio de Golofa (Morón \& Pardo-Locarno, 1994), en el marco del proyecto Coleópteros del Calima (Colciencias 2108-05-005, 1989-1993). Posteriormente, en febrero de 1998 continuó con el apoyo de Patrimonio Ambiental de la Corporación Autónoma Regional del Valle del Cauca (CVC) (OTGVS 038; PardoLocarno, 1998). Fases siguientes de ciclos de vida se culminaron entre 1998 y 2003 con el apoyo del IIAP. Entre enero y diciembre de 2005 se complementaron aspectos biológicos y técnicos con el convenio CVG y el Centro para la Investigación en Sistemas Sostenibles de Producción Agropecuaria (CIPAV) (PardoLocarno, 2006). Algunos temas biológicos se subsanaron entre 2010 y 2012 (convenio CVC-AAFP 218-2010). Múltiples actividades biométricas y de laboratorio fueron realizadas entre 2011 y 2017. El informe final se realizó como iniciativa de diversas instituciones, entre ellas la Universidad del Pacífico y Corpoamazonía.

\section{Resultados y Discusión}

A continuación, se describen las larvas de tercer instar, pupas y datos biológicos (ciclos de vida) de D. h. lichyi Lachaume, D. $h$. occidentalis Lachaume y de Dynastes neptunus Quenzel (Coleoptera: Melolonthidae: Dynastinae: Dynastini), al final se presentan claves para larvas y pupas de Dynastes y una corta discusión de resutados.

\section{Descripción de inmaduros de Dynastes hercules L 1758}

Dynastes hercules lichyi Lachaume $\mathbf{1 9 9 5}$, descripción de la larva de tercer instar (Figuras 1, 2, 3, 4 y 5).

Basado en: tres larvas de tercer instar fijadas en 2003 y 2004 obtenidas de la cría de inmaduros con parentales colectados en La Paz (Santander - Colombia) en abril de 2002; 4-5 exuvias de larvas de tercer instar, misma procedencia, fijadas en 2004.

La longitud de los ejemplares medida en larvas vivas fue de entre 90 y $110 \mathrm{~mm}$ (Figura 1A). Cabeza: el rango de anchura cefálica fue de entre 14 y18 mm (Figura 2A). Color: pardo oscuro rojizo, superficie densa y profundamente rugosa punturada, punturas 

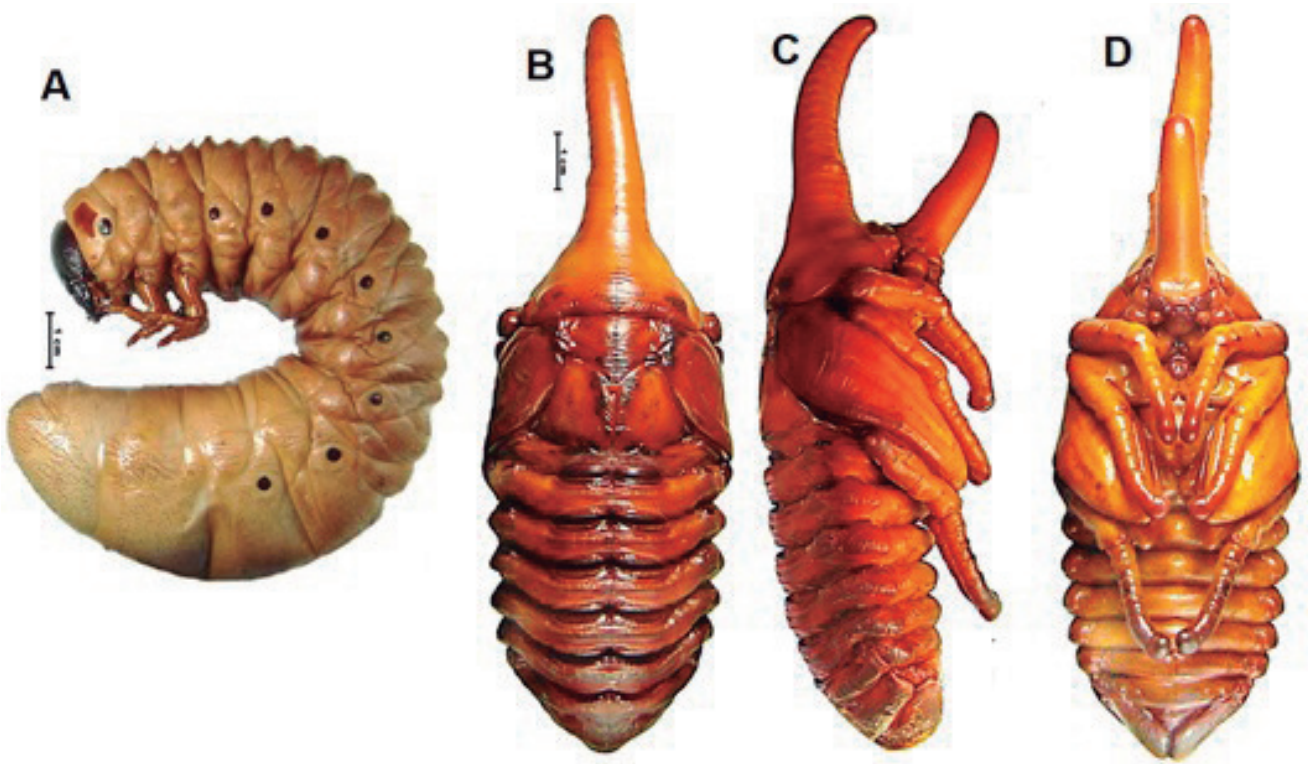

Figura 1. Dynastes hercules lichy: A: larva tercer instar; B, C, y D pupa dorsal, lateral y ventral, respectivamente.

profundas, separadas por una o dos veces su diámetro, a veces muy unidas o coalescentes. Frente con una seta posterior frontal y una a dos setas exteriores frontales a cada lado, dos pequeñas setas anteriores frontales a cada lado; ángulo anterior de la frente con dos a tres setas; resto de la cabeza con cuatro a cinco setas dorsoepicraneales, de ocho a diez setas paraocelares y basiantenales a cada lado; precoila pigmentada; stemmata visibles color ámbar claro; clípeo con dos setas centrales y dos setas laterales; labro asimétrico, con bordes laterales ampliamente curvados; al ápice con una muesca sutil en su porción centro derecha y unas 10 a14 setas apicales. Epifaringe (Fig. 2B) con proceso haptomeral prominente, oblicuo al plano longitudinal; acanthoparia con 12 a 14 setas robustas curvas, las setas finales menos curvadas y contiguas a la acroparia; gymnoparia corta, más ensanchada en su porción central; plegmatia ausente; chaetoparia derecha con 18 a 22 sensilas en el borde externo y 38 a 40 setas robustas en torno al pedium, el cual es largo y ovalado; chaetoparia derecha similar pero con 24 a 30 setas más robustas en torno al pedium; acroparia con seis a ocho setas robustas; corypha poco dotada; dexiotorma corta angostada al ápice; laeotorma larga; pternotorma pigmentada, globular; placa esclerosada subtriangular, aguzada, pigmentada; cono sensorial largo, en forma de barra, con ápice cupuliforme; crepis suavemente pigmentado.

Mandíbula izquierda (Figura 3A) dotada de dos incisivos, largos los cuales están 

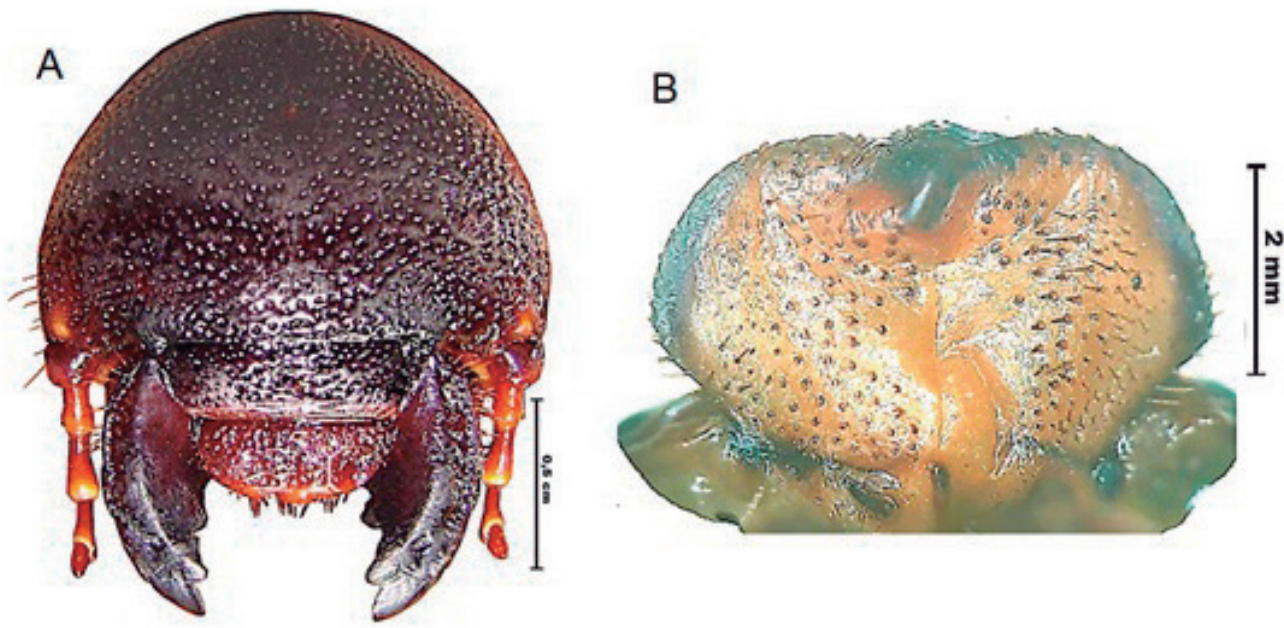

Figura 2. Dynastes hercules lichy: A: Cabeza; B: Epifaringe

separados por una muesca obtusa, seguido de otra muesca ensanchada y otro diente corto redondeado. En el ápice mandibular se observa una seta dorsal, larga; área molar conformada por tres coronas, la primera grande y prominente, las dos siguientes cortas y contiguas; acia larga, ápice agudo; brustia pequeña; área estriduladora mandibular larga, ovalada, inicialmente con 12 a 14 pliegues anchos y luego 14 a 18 pliegues muy angostos parecidos a líneas; puente ventral grande (Figura 3B);
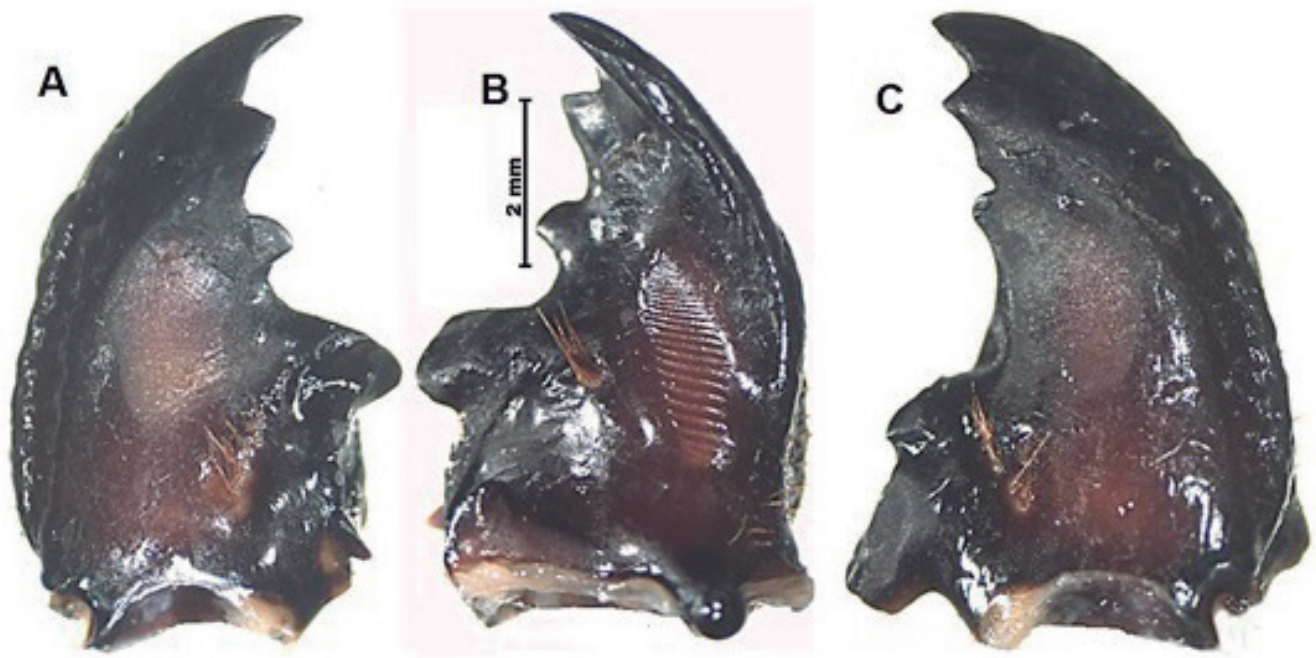

Figura 3. Dynastes hercules lichy: A: Mandíbula izquierda dorsal; B: Mandíbula izquierda ventral; C: Mandíbula derecha dorsal. 
mandíbula derecha (Figura 3C) con área incisiva muy similar, región media inerme seguida del área molar conformada por M1 grande y las siguientes coronas bajas y unidas; calx proyectado; brustia reducida; área estriduladora similar; en ambos casos scrobis largo, profundo, apicalmente dotado de dos setas separadas.

Labium (Figura 4B), disco rodeado de 38 a 40 setas basales, 18 a 20 setas en el borde lateral y 12 a 14 setas en la porción apical entre los palpos labiales; escleroma hipofaríngeo asimétrico con una proyección aguda a la derecha y 18 a 20 setas en el lóbulo lateral izquierdo y 12 a 14 en el derecho. Maxila (Figura 4A) conformando una mala, con un uncus prominente en la galea y tres unci apicales en la lacinia, estos unidos en su base; borde interno de la lacinia densamente setoso; palpo maxilar con el tercer segmento dotado de una seta en el borde externo; en la porción dorsal se observan seis a siete dientes estriduladores que culminan en un proceso truncado más grande y oblicuo. Antenas largas, robustas (Figura 4B), con el área dorsal del segmento apical dotado de 12 a 15 fosetas sensoriales (Figura 4C).

Tórax: espiráculo torácico grande, aprox. $2.1 \mathrm{~mm}$ de alto y $1.8 \mathrm{~mm}$ de ancho (Figura $4 \mathrm{D})$; placa respiratoria pardo rojiza en forma de C, con los ápices contiguos; bulla

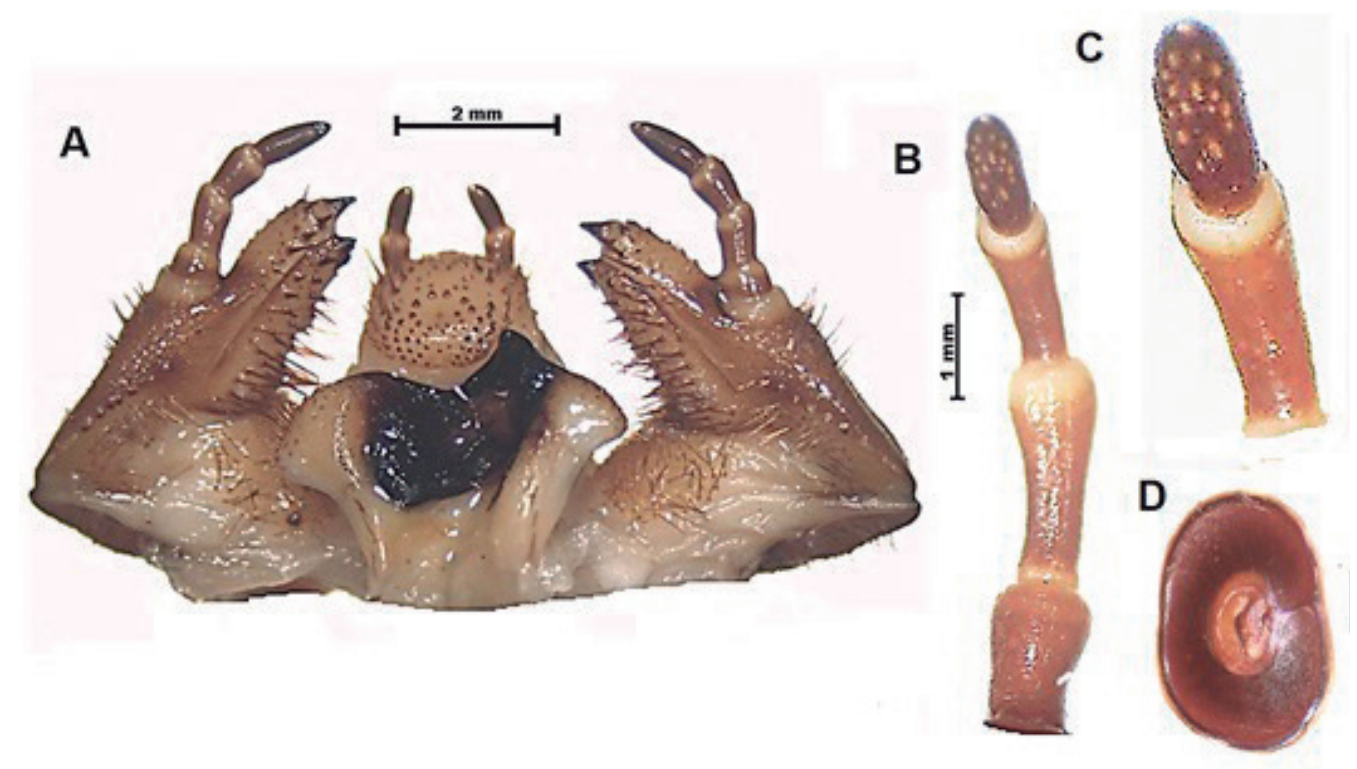

Figura 4. Dynastes hercules lichy: A: Labium-Maxilas; B: Antena; C: Últimos dos segmentos antenales, vista dorsal; D: Estigma respiratorio protórax. 
pequeña levemente convexa; escleroma lateral largo, sub romboidal, con el disco semisurcado, cóncavo y algo pigmentado (Figura 1A); placas dorsales del protórax y mesotórax poco diferenciadas, con múltiples setas cortas espiniformes; placa dorsal del metatórax con bandas más definidas de setas cortas espiniformes y algunas setas largas; patas grandes y robustas, densamente setosas, las pro y mesotorácicas con la uña aguzada y dos setas alternas, una basal y otra apical; uña tarsal de las patas metatorácicas no aguzada, con dos setas subpicales (Figura 5A y 5 B).

Abdomen: espiráculos abdominales I-VIII (Figura 1A) de tamaño similar (1.9 mm a 2.0 $\mathrm{mm}$ de alto y $1.7 \mathrm{~mm}$ a $1.8 \mathrm{~mm}$ de ancho), con placa respiratoria en forma de $\mathrm{C}$ y ápices contiguos (Figura 4D); bulla exiguamente proyectada; dorsa de los segmentos I-VI con cada placa (prescutum, scutum y scutellum) claramente definida, dotadas de múltiples setas cortas espiniformes y en menor grado setas largas en franjas posteriores; dorsa de los segmentos VII y VIII con placa única, dotada de bandas de múltiples setas cortas espiniformes (2030) y unas pocas setas largas en el borde posterior (entre 16 y 18); dorsa IX similar a la VIII; venter I-IX dotado de franjas de 20 a 30 setas cortas espiniformes y cinco setas largas; ráster (Figura 5C) sin palidia, campus con pocas setas mixtas, teges con una formación triangular de 20 a 30 setas cortas espiniformes y unas 6 a 8 setas largas; labio anal inferior con una centena de setas cortas espiniformes, barbula con 18 a 20 setas largas; abertura anal larga, poco curvada.
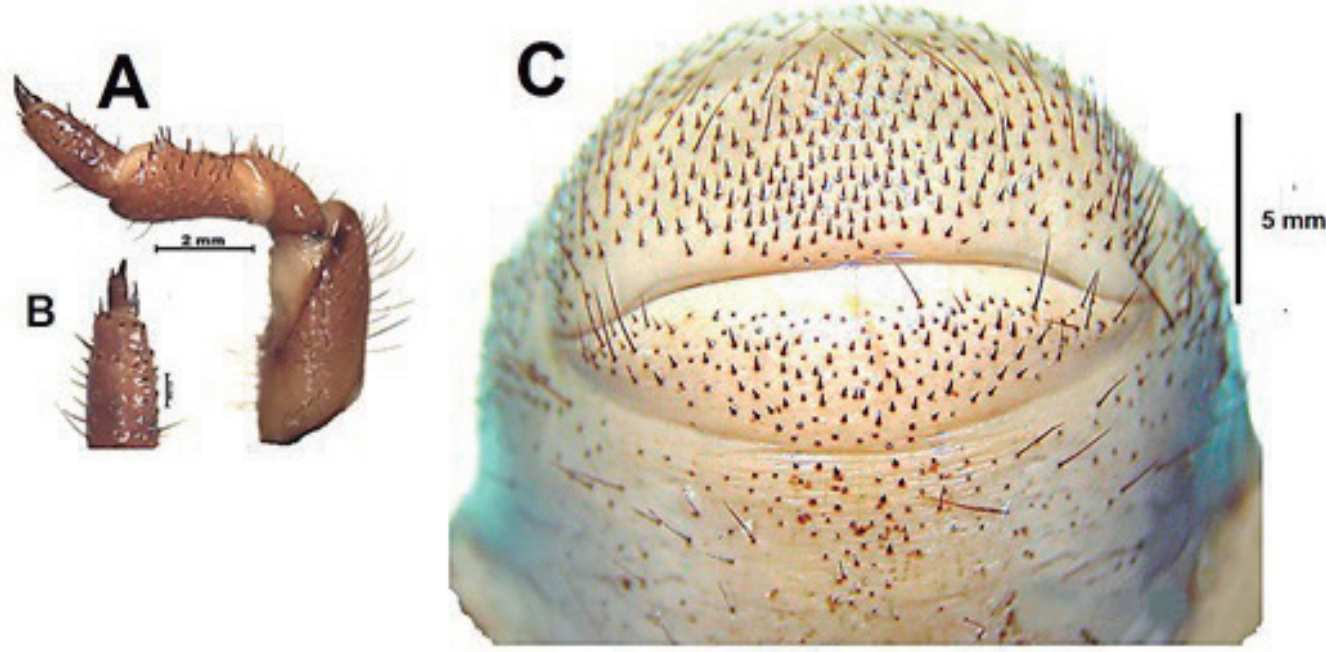

Figura 5. Dynastes hercules lichy: A: Patas protorácicas; B: Tarsunguli-uña; C Abdomen, ráster. 
Dynastes hercules lichyi Lachaume, descripción de la pupa (Figuras $1 \mathrm{~B}, 1 \mathrm{C}$ y 1D).

Basado en: tres pupas ( $1 \hat{\widehat{O}} \mathrm{y} 2$ q $q$ ) fijadas en 2003 y 2004 obtenidas de la cría de inmaduros con parentales colectados en La Paz (Santander - Colombia) en abril de

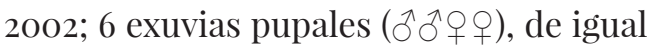
procedencia, fijadas entre 2004 y 2005.

Longitud corporal entre 78 y $128 \mathrm{~mm}$. Macho (Figuras 1: B, C y D). Exarata, cuerpo robusto, ovalado, color amarillo oscuro, algo rugoso y setas ubicadas en el ápice abdominal; cabeza ubicada en posición ventral al protórax (Figura 1C), región frontal con una larga proyección central y bosquejo de dos oquedades rugosas a los lados; con estructuras bucales y sensoriales visibles: clípeo transverso, en forme de barra, labro ubicado centralmente bajo el clípeo; ápices mandibulares y antenales visibles, palpos maxilares y labiales con anillos visibles (Figura 1D); ojos casi ocultos; labium constreñido por los palpos; Tórax, pronotum transverso, largamente proyectado por el cuerno respectivo (Figura 1B), que en este caso es el doble de largo que el protórax; borde anterior sinuado, con proyecciones laterales que rodean la cabeza, borde posterior levemente bisinuado con los ángulos posteriores redondeados; mesotórax dorsalmente visible en un escudo cuyo borde anterior es recto y el posterior centralmente proyectado; sutura ecdisial longitudinal visible; proceso proesternal visible y proyectado en forma de disco; mesosternum oculto parcialmente bajo las podo y pterotecae; metanoto anteriormente biescotado, borde posterior casi recto, levemente situado y surco ecdisial longitudinal visible; pterotecae mesotorácicas dorsal y lateralmente visibles y muy proyectadas, las pterotecae metatorácicas ocultas y solo visibles al ápice; podotecae bastante visibles, largas, las protorácicas dirigidas hacia la región esternal, las meso y metatorácias hacia los lados y atrás, dejando ver a los extremos los anillos tarsales.

Abdomen, con nueve segmentos (Figuras 1B, 1C y 1D), basalmente más anchos; espiráculo I presente, oculto bajo el borde posterior torácico (observado en un exuvia pupal); espiráculos II, III y IV visibles dorsalmente, en todos los casos con los peritremas claramente pigmentados, ovalados, aprox. 3 veces más largo que ancho (aprox. 8.5 $\mathrm{mm}$ por 1.9 a $2.0 \mathrm{~mm}$ ); espiráculos V, VI y VII ocluidos, vestigiales; espiráculo VIII no visible; región tergal de los segmentos I-VII con sendos órganos dioneiformes, pareados, aprox. 17-18 mm por 2-3 mm; órgano dioneiforme VI no pareado, porción proximal no definida, la porción posterior bien formada; segmento IX aguzado, triangular, apicalmente bifurcado, con una densa cobertura setosa dorada; esternito I oculto bajo las podotecae; esternitos II, III, IV, V, VI y VII muy cortos en forma de franjas convexas, con ápices laterales en forma de muñones; esternitos VIII y IX aguzados, en la región ventral dejan ver el ámpula genital masculina, en forma de disco convexo con una formación cilíndrica levemente trazada.

Hembra: longitud medida en seis pupas de entre $68 \mathrm{~mm}$ y $78 \mathrm{~mm}$; muy similar a la del 
macho, cabeza ubicada en posición ventral al protórax, inerme, frente con sendas oquedades a cada lado; con estructuras bucales y sensoriales apicalmente visibles; tórax, pronotum convexo, inerme, con una corta proyección en forma de tubérculo; abdomen con los espiráculos II, III y IV visibles, bien formados; los siguientes V, VI y VII ocluidos, vestigiales, carentes de peritrema; órganos dioneiformes en los segmentos I a VII, en I a VI pareados, en VII no pareado, en todos los casos cada dorsa presenta una angosta banda grisácea transvera; dorsa de terguito VIII con dos oquedades laterales, difusas; ámpula genital levemente convexa, con bosquejo de valvas genitales.
Aspectos biológicos: a fin de ahondar en una próxima entrega los detalles del ciclo de vida de D. h. lichyi (Pardo-Locarno In litt), se comparten por ahora referentes puntuales en las tablas 1 y 2; en general los datos obtenidos presentaron alguna similitud con los expuestos por Gruner $\&$ Chalumeau (1979) para D. h. hercules y con lo anotado por Morón (1987) para $D$. hyllus Chev., los cuales variaron entre 1.9 y 2.3 años; el ciclo de vida de $D$. $h$. lichyi, desarrollado en condiciones de laboratorio, ex situ, vario de 1.4 a 2.4 años, con una media de 14-16 meses, resaltaron algunos pocos individuos que se demoraron el doble de tiempo (Tabla 1).

Tabla 1. Ciclos de vida en cría ex situ de D. h. lichyi, D. h. occidentalis y D. neptunus, duración en días (abreviaturas: L1: larva I, primer estado; L2: larva II, segundo estado;

L3: larva III, tercer estado; PP prepupa; P: pupa; N: cantidad de observaciones; + : la cifra es un poco mayor; * ver comentarios para más explicaciones).

\begin{tabular}{ccccccccc}
\hline $\begin{array}{c}\text { Especie/ } \\
\text { atributo }\end{array}$ & \multicolumn{7}{c}{ Dynastes hercules lichy Lachaume } \\
\hline Fase & Huevo & L1 & L2 & L3 & PP & P & Adulto & Rango \\
\hline Duración fase (D) & $28-35$ & $28.5-33.5$ & $47-96.5$ & $320.5-425.5$ & $11-28$ & $36-50$ & $42-135$ & $513-899$ \\
\hline N datos & 11 & 11 & 11 & 9 & 8 & 6 & 6 & \\
\hline Especie & \multicolumn{7}{c}{ Dynastes hercules occidentalis Lachaume } \\
\hline Fase & Huevo & L1 & L2 & L3 & PP & P & Adulto & Rango \\
\hline Duración fase (D) & $25-34$ & $70-97$ & $78-93$ & $465-607$ & $21-24$ & $28-35$ & $95-178$ & $540-720$ \\
\hline N (datos) & 10 & 4 & 2 & 2 & 2 & $2^{*}$ & $6^{*}$ & $2 *$ \\
\hline Especie & & \multicolumn{7}{c}{ Dynastes neptunus Quenzel } \\
\hline Fase & Huevo & L1 & L2 & L3 & PP & P & Adulto & Rango \\
\hline Duración fase (D) & $29.5-35.5$ & $47-97$ & $30-121$ & $277-413$ & $31-35$ & $29-40$ & $90-156$ & $533-915$ \\
\hline N datos & 20 & 18 & 12 & 9 & 9 & $8^{*}$ & $5^{*}$ & \\
\hline
\end{tabular}


Estas observaciones ratifican lo dicho por otros autores respecto a la mayor vulnerabilidad de los estados de huevo y larva de primer instar, asimismo, la extensión del estadio de tercer instar; la pupa se protegió en un cocón construido con residuos de madera y algún exudado secretado por la larva, lo cual le confirió a la estructura una relativa estabilidad; los adultos tenerales evidenciaron un color claro y demoraron entre 4 y 5 días para lograr una pigmentación normal, en ese tiempo produjeron una primer excreta o meconio más bien acuoso; el deseo de alimentarse se observó entre los 8 y 10 días en adelante, entre 18 y 20 días se evidenciaron las primeras interacciones de pareja.

Relativo al larguo periodo de inmaduros, los adultos tuvieron una longevidad corta, algo que fue más marcado en los machos; las hembras por lo regular duraron el doble del tiempo, en promedio entre dos y tres meses, sobrepasando algunas los cuatro meses. Entre las medidas corporales tomadas (Tabla 2) se confirmó en primer lugar la elongación de corion en el estado de huevo, pasando este de la forma elíptica a la esférica y al final exponiendo vagamente el pigmento de la cabeza y los segmentos de la larvita en su interior. Dichas medidas dejan claro a la cabeza como la medida de crecimiento con los datos más discretos, variando entre 3.6 a $4.3 \mathrm{~mm}$ (LI) hasta 13 a $17 \mathrm{~mm}$ (LIII), lo cual esboza una media de ampliación de la anchura cefálica de 1.8 a 1.9 veces entre cada estadio, algo que se asemeja a lo medido en D. hyllus (Morón, 1987).
Tabla 2. Medidas corporales (mm) de los estadios de desarrollo y adultos parentales de la cría ex situ de D. h. lichyi, D. h. occidentalis y D. neptunus (abreviaturas: Ø' huevo recién puesto; $\varnothing^{2}$ huevo elongado; Li: larva I, primer estado; L2: larva II, segundo estado;

L3: larva III, tercer estado; PP prepupa;

P: pupa; N: cantidad de observaciones.

\begin{tabular}{|c|c|c|c|}
\hline $\begin{array}{c}\text { Especie/ } \\
\text { estadio }\end{array}$ & $\begin{array}{c}\text { Ancho } \\
\text { cefálico }\end{array}$ & $\begin{array}{l}\text { Ancho } \\
\text { cuerpo }\end{array}$ & Longitud \\
\hline Especie & \multicolumn{3}{|c|}{ Dynastes hercules lichy Lachaume } \\
\hline Huevo & $\boldsymbol{\emptyset}^{1}=$ & $\emptyset^{2}=$ & \\
\hline Larva 1 & $3.6-4 \cdot 3$ & $3.9-18$ & $15-73$ \\
\hline Larva 2 & $6.4-9$ & $10-26$ & $31-115$ \\
\hline Larva 3 & $13-17$ & $19-44$ & $80-185$ \\
\hline Prepupa & $13-17$ & $36-42$ & $82-1$ \\
\hline Pupa ${ }_{+}$ & 10.5 & $32-35$ & $68-78$ \\
\hline Pupa $\widehat{\sigma}$ & 10.5 & $38-44$ & $78-128$ \\
\hline Adulto ${ }_{+}$ & & & $155^{+}$ \\
\hline Adulto $\lesssim$ & & & \\
\hline
\end{tabular}

\begin{tabular}{cccc}
\hline Especie & \multicolumn{3}{c}{ Dynastes hercules occidentalis } \\
Lachaume \\
\hline Huevo & $\boldsymbol{\emptyset}^{1=3.0}$ & $\boldsymbol{\emptyset}^{2}=5-6$ \\
\hline Larva 1 & $3.2-3.5$ & $2.5-4.0$ & $10-22$ \\
\hline Larva 2 & $6.5-7$ & $6.0-9.0$ & $30-40$ \\
\hline Larva 3 & $11-11-5$ & $12-22$ & $45^{-90}$ \\
\hline Prepupa & 11.5 & 21 & 80 \\
\hline Pupa + & 11.5 & 28.5 & $66-67^{+}$ \\
\hline Pupa 0 & 12 & 30 & $78-79^{+}$ \\
\hline Adulto + & & & $168+$ \\
\hline Adulto & & & \\
\hline
\end{tabular}




\begin{tabular}{|c|c|c|c|}
\hline $\begin{array}{c}\text { Especie/ } \\
\text { estadio }\end{array}$ & $\begin{array}{l}\text { Ancho } \\
\text { cefálico }\end{array}$ & $\begin{array}{l}\text { Ancho } \\
\text { cuerpo }\end{array}$ & Longitud \\
\hline Especie & \multicolumn{3}{|c|}{ Dynastes neptunus Quenzel } \\
\hline Huevo & $\boldsymbol{D}^{1}=4.5$ & $\boldsymbol{O}^{2}=6.0$ & \\
\hline Larva 1 & $3.9-4.1$ & $6.3-9.3$ & $27-44.9$ \\
\hline Larva 2 & $6.4-7.8$ & $11-16.5$ & $55.5-90$ \\
\hline Larva 3 & $12.5^{-13}$ & $19-32$ & $86-142$ \\
\hline Prepupa & $11-13$ & 22 & 112 \\
\hline Pupa ${ }_{+}$ & 11 & 28 & 52 \\
\hline Pupa $\widehat{\sigma}$ & 12.8 & 33 & 76 \\
\hline Adulto $q$ & - & - & $145^{+}$ \\
\hline Adulto $\lesssim$ & - & - & \\
\hline
\end{tabular}

Otro aspecto que evidenció datos discretos fue el tamaño de las excretas (Figura 16A), en ese sentido los pelets de tercer instar midieron en promedio 15 a $18 \mathrm{~mm}$ de largo y entre 12 y 14 de ancho; para futuros ensayos bien valdría la pena atender las sugerencias de Dupuis \& Dubois (1997) sobre el enriquecimiento de la dieta larvaria con glúcidos y proteínas, como un factor determinante de menores tiempos de cría y quizás, individuos más grandes y saludables. Cabe anotar que, a pesar de la gran diversidad de páginas web halladas, este artículo sería de los pocas en desarrollar el ciclo de vida de $D$. h. lichyi, formalmente, en una publicación de carácter científico.

El estudio de Huang (2017) sobre el subgénero Dynastes, que erige múltiples subespecies a rango especifico, basado en modelos moleculares, feneticistas, geográficos, ecológicos, etc., ratifica una evidente divergencia intraespecífica, pero sin el alcance suficiente para establecer estatus específicos, máxime con la precaria información geográfica, ecológica etc. que caracteriza a la subespecie, dejando de lado además el estudio de barreras reproductivas, que evidencien imposibilidad de cruce exitoso, lo cual sería, el sello final para afirmar la conformación de especies.

Sobre la distribución real de la subespecie en Colombia, aún existen muchos vacíos (Pardo-Locarno et al., 2020); no obstante, existe consenso respecto a que está muy asociada a la Cordillera Oriental, en especial a la cuenca media y baja del Río Magdalena (Lachaume, 1994; PardoLocarno et al., 2020). Ballesteros \& AmatGarcía (2006), estudiaron la abundancia de la subespecie en dos hábitats típicos: robledal y selva de lauráceas, al final observaron que la oferta ambiental en el primero fue mejor en cuanto a troncos descompuestos y frutos, pero la abundancia fue mayor en el segundo, debido a la menor caza de escarabajos; sin embargo, este informe no publicó los referentes geográficos del estudio. Ardila \& Palomino (2017) también estudiaron en campo la presencia de adultos, ofreciendo datos de localidad (La Belleza, Santander, Valle del Río Magdalena), igualmente afectados por el problema de la extracción ilegal. A la fecha ninguna investigación ha llevado a cabo un estudio de campo específico, amplio y suficiente para establecer el verdadero mapa de la subespecie en Colombia (PardoLocarno et al., 2020); mientras ello no 
ocurra, persistirán muchos vacíos en el conocimiento cabal de la misma.

\section{Dynastes hercules occidentalis \\ Lachaume 1995, redescripción de larva \\ de tercer instar (Figuras 6, 7, 9, 10).}

Basado en dos exuvias de larvas de tercer instar fijadas el 25 de octubre de 2014, obtenidas de la cría de inmaduros con parentales colectados en La Quebrada Triana, Bajo Dagua (Valle del Cauca Colombia), el 26 de junio de 2011 y una larva de tercer instar, con los mismos datos. La descripción atendió lo descrito por Villamarín-Cortez et al (2007) para D. h. occidentalis Lachaume en Ecuador.

En general, la larva de tercer instar de esta subespecie guarda gran similitud con D. h. lichyi. La longitud de los ejemplares, tomada en larvas vivas fue de entre $90 \mathrm{~mm}$ y $110 \mathrm{~mm}$ (Figura 9C). Cabeza: la anchura cefálica fue de entre $12.2 \mathrm{~mm}$ y $13 \mathrm{~mm}$ (Figura $7 \mathrm{~A}$ ); color pardo oscuro, con un tinte rojizo; superficie densa y profundamente rugosa punturada, punturas profundas, separadas por una o dos veces su diámetro, a veces unidas o coalescentes; frente con una a dos setas exteriores frontales a cada lado, dos pequeñas setas anteriores frontales a cada lado; ángulo anterior de la frente con dos a tres setas; resto de la cabeza con dos a cuatro setas dorsoepicraneales; un grupo de setas paraocelares y basiantenales, precoila pigmentada; stemmata visibles de color ámbar claro; clípeo con dos setas centrales y dos-tres setas laterales a cada lado; labro asimétrico apicalmente con una muesca sutil en su porción centro derecho y de 10 a 14 setas apicales. Epifaringe (Figura 6D) con proceso haptomeral prominente, oblicuo al plano longitudinal; acanthoparia con 12 o más setas curvas, las finales casi rectas y próximas a la acroparia; gymnoparia larga, ensanchada en su porción central; plegmatia ausente; chaetoparia derecha con 18 a 22 sensilas en el borde externo y entre 38 y 40 sensilas, más robustas en el interno, chaetoparia derecha similar pero con 24 a 30 setas más robustas en torno al pedium, el cual es largo y ovalado; dexiotorma larga angostada al ápice; laeotorma larga; pternotorma pigmentada, globular; placa esclerosada subtriangular pigmentada; cono sensorial largo, en forma de barra con el ápice redondeado; crepis suavemente pigmentado.

Mandíbula izquierda (Figura 6A), dotada de dos incisivos largos los cuales están separados por una muesca obtusa; segunda proyección incisiva seguida por un espacio corto y un diente corto, triangular, sigue un área inerme corta para dar paso a los molares; área molar conformada por tres coronas, la primera grande y prominente, las dos siguientes cortas y contiguas; brustia pequeña; área estriduladora mandibular larga, ovalada, inicialmente con 12 a 14 pliegues anchos y 14 a 18 pliegues angostos; puente ventral grande; mandíbula derecha (Figura 6B) con área incisiva constituida por tres proyecciones triangulares, la dos y tres separadas por una muesca triangular, sigue un espacio y luego un diente más corto, luego un espacio inerme cóncavo seguido del área molar, conformada por una sola corona molar irregular (que engloba M1, M2 y M3); en ambos casos scrobis largo, profundo, punturado y con seta apical. Labium setoso, 
con escleroma hipofaríngeo asimétrico, con una proyección aguda a la derecha. Maxila conformando una mala, con un uncus prominente en la galea y tres unci apicales en la lacinia, palpo maxilar con el tercer segmento dotado de una seta en el borde externo; en la porción dorsal se observan seis a siete dientes estriduladores, que culminan en un diente truncado más grande y oblicuo. Antenas largas, robustas, con el área dorsal del segmento apical dotado de entre 10 y 12 fosetas sensoriales.
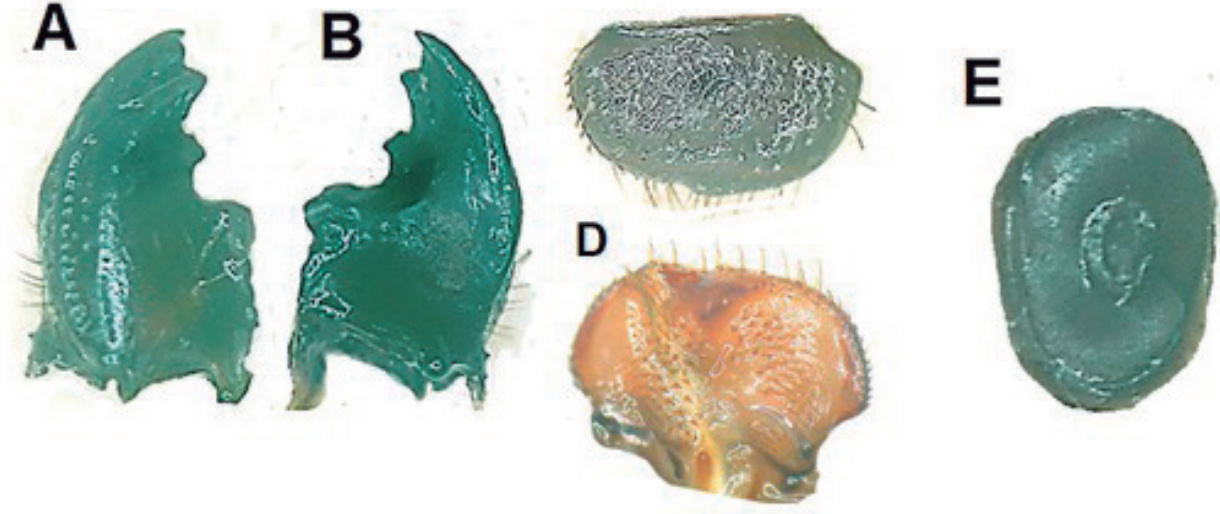

Figura 6. Dynastes hercules occidentalis: A: Mandíbula izquierda dorsal; B: Mandíbula derecha dorsal; C: Labro; D: Epifaringe; E: Estigma respiratorio protórax.

Tórax: espiráculo torácico grande, aprox. 2.0 $\mathrm{mm}$ de alto y $1.5 \mathrm{~mm}$ de ancho (Figura 6E); placa respiratoria pardo rojiza en forma de C con los ápices contiguos; bulla pequeña poco convexa, alargada; escleroma lateral largo, subromboidal, con el disco cóncavo y pigmentado; placas dorsales del protóraxy mesotórax poco diferenciadas con múltiples setas cortas espiniformes; placa dorsal del metatórax con bandas más definidas de setas cortas espiniformes y algunas setas largas; patas robustas, multisetosas, último segmento con la uña aguzada y dos setas alternas, una basal y otra apical; uña tarsal de las patas metatorácicas no aguzada, con dos setas subpicales.

Abdomen: espiráculos abdominales I-VIII (Figura 9C) de tamaño similar (1.8 $\mathrm{mm}$ de alto y $1.4 \mathrm{~mm}$ de ancho), placa respiratoria en forma de C y ápices contiguos; bulla exiguamente proyectada; dorsa de los segmentos I-VI con cada placa (prescutum, scutum y scutellum) claramente definida, dotadas de múltiples setas cortas espiniformes y en menor grado, setas largas en la franja posterior; dorsa de los segmentos VII y VIII con placa única, 
dotada de bandas de múltiples setas cortas espiniformes y unas pocas setas largas en el borde posterior; dorsa IX similar a VIII; ráster sin palidia (Figura $7 \mathrm{~B}$ ), teje en formación triangular con 20 a 30 setas cortas espiniformes y algunas setas largas; labio anal inferior densamente setoso, principalmente setas cortas espiniformes; abertura anal larga, poco curvada.
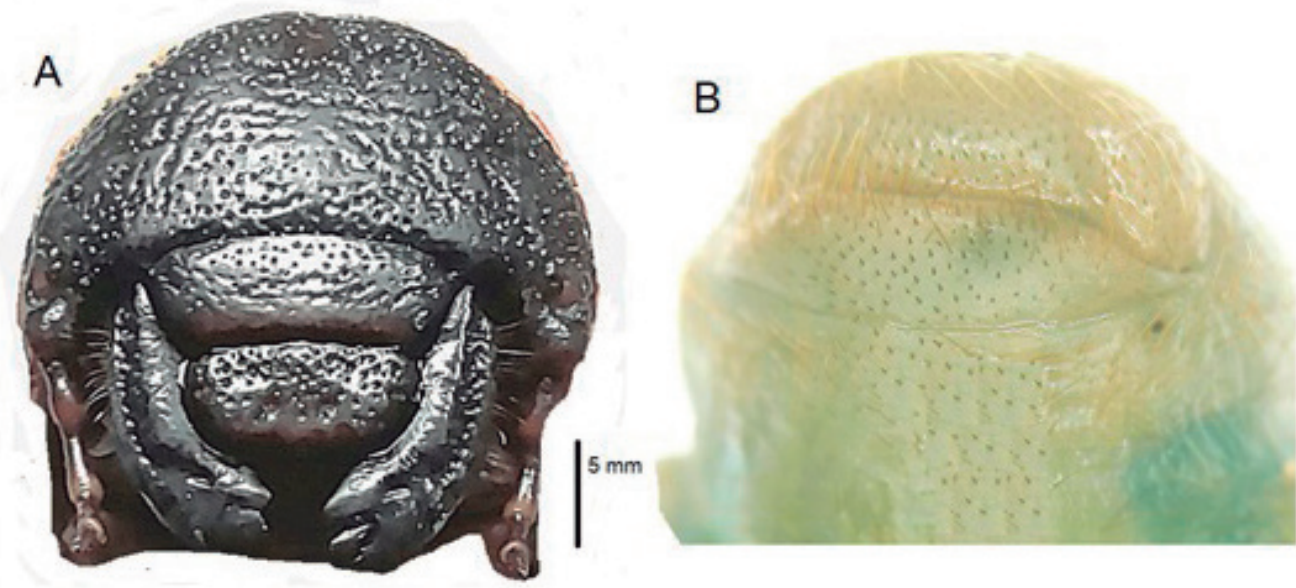

Figura 7. Dynastes hercules occidentalis: A: Cabeza; B: Abdomen, ráster.

\section{Dynastes hercules occidentalis Lachaume 1995, descripción de la pupa (Figuras 8,9 )}

Basado en dos pupas (una masculina y otra femenina) fijadas el 25 de octubre de 2014, obtenidas de la cría de inmaduros con parentales colectados en la Quebrada Triana, Bajo Dagua (Valle del Cauca Colombia), el 26 de junio de 2011.

La longitud corporal medida en una sola pupa fue de entre 78 y $79 \mathrm{~mm}$. en el macho (Figura 8). Exarata, cuerpo robusto, ovalado, color amarillo oscuro, algo rugoso y setas ubicadas al ápice abdominal; cabeza ubicada en posición ventral al protórax (Figura 8B, 8C), región frontal con una proyección cefálica y dos borrosas y rugosas oquedades laterales; con estructuras bucales y sensoriales visibles: clípeo transverso en forma de barra, labro ubicado centralmente bajo el clípeo (Figura 8B, 8C); ápices mandibulares y antenales visibles, palpos maxilares y labiales con anillos visibles; ojos casi ocultos; labium constreñido por los palpos; tórax, pronotum transverso, dotado de cuerno (Figura 8A); borde anterior sinuado, con proyecciones laterales que rodean la cabeza, borde posterior levemente bisinuado con 
los ángulos posteriores redondeados; mesotórax dorsalmente visible en un escudo cuyo borde anterior es recto y el posterior centralmente proyectado; sutura ecdisial visible; proceso proesternal visible y proyectado en forma de disco (Figura $8 \mathrm{~A}$ ); mesosternum oculto parcialmente bajo las podo y pterotecae (Figura 8C); metanoto largo, anteriormente biescotado, borde posterior casi recto, levemente situado y surco ecdisial longitudinal visible; pterotecae mesotorácicas dorsal y lateralmente visibles y muy proyectadas, las pterotecae metatorácicas ocultas y solo visibles al ápice (Figura 8C); podotecae bastante visibles, largas, las protorácicas dirigidas hacia la región esternal, las meso y metatorácicas hacia los lados y atrás, dejando ver a los extremos los anillos tarsales (Figura 8B y 8C).

Abdomen (Figura 8A y 8C), con nueve segmentos basalmente más anchos; espiráculo I presente, oculto bajo el borde posterior torácico (observado en un exuvia pupal); espiráculos II, III y IV visibles dorsalmente (Figura 8C), en todos los casos con los peritremas pigmentados, ovalados, casi tres veces más largo que ancho (aprox. entre $8.5 \mathrm{~mm}$ y 1.9 a $2 \mathrm{~mm}$ ); espiráculos sobre terguitos V, VI y VII ocluidos, vestigiales, carentes de peritrema esclerosado, VIII bastante vestigial poco visible; región tergal de
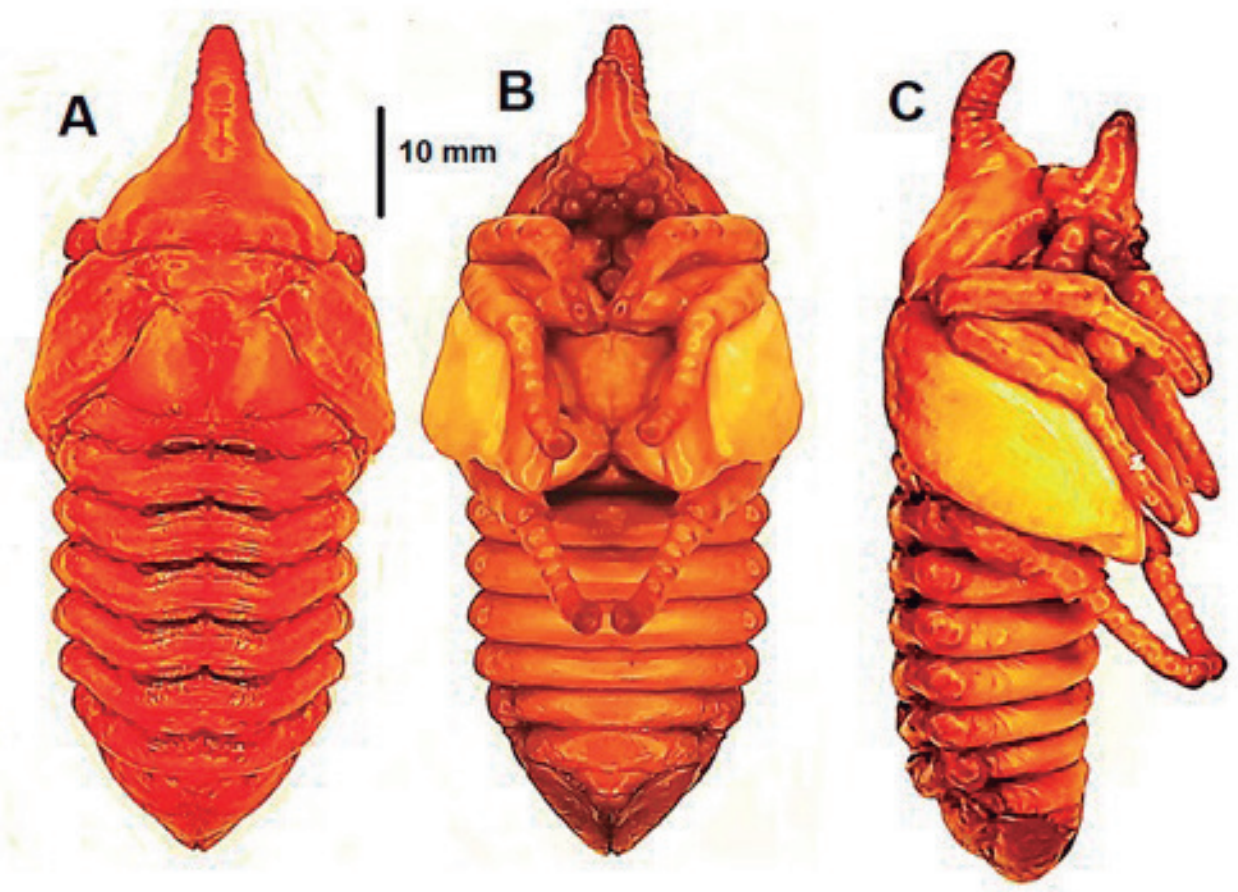

Figura 8. Dynastes hercules occidentalis: Pupa macho: A: dorsal; B: ventral; C: lateral. 
los segmentos I-VII con seis pares de órganos dioneiformes (Figura 3C); órgano dioneiforme VI no pareado (Figura 8C), porción proximal no definida, la porción posterior bien formada; terguito IX con dos oquedades laterales un poco alargadas y algo difusas; segmento IX aguzado, triangular, apicalmente bifurcado, con una densa cobertura setosa dorada; esternito I oculto bajo la podotecae; esternitos II, III, IV, V, VI y VII muy cortos en forma de franjas convexas, con ápices laterales en forma de muñones; esternitos VIII y IX aguzados, en la región ventral dejan ver el ámpula genital masculina, muy levemente figurada, en forma de disco convexo.

Hembra (Figura 9A, 9B). Longitud medida en una pupa de entre $66 \mathrm{~mm}$ y $67 \mathrm{~mm}$, anchura máxima del tórax entre $29 \mathrm{~mm}$ y
$30 \mathrm{~mm}$. En general muy similar al macho, excepto por algunos rasgos genéricos; cuerpo robusto, ovalado, color amarillo oscuro, algo rugoso, setas ubicadas al ápice abdominal (Figura 4A); cabeza inerme, ubicada en posición ventral al protórax (Figura 9A); con estructuras bucales y sensoriales visibles similares a las del macho; frente con sendas oquedades a cada lado; Tórax, pronotum convexo, inerme, con una corta proyección en forma de tubérculo; abdomen con los espiráculos II, III y IV visibles, bien formados (Figura 9B); los siguientes V, VI y VII ocluidos, vestigiales, carentes de peritrema (Figura 9A); órganos dioneiformes en los segmentos I a VII, en I a VI pareados (Figura 9A), en VII no pareado (Figura 9A); ámpula genital levemente convexa, con bosquejo de valvas genitales (Figura 9B).
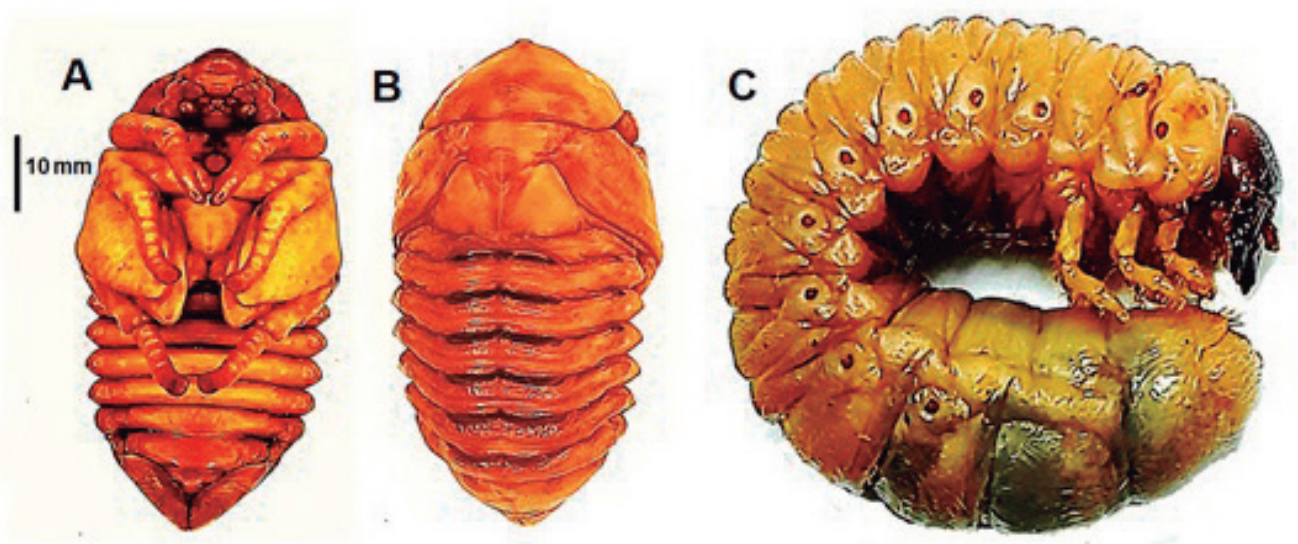

Figura 9. Dynastes hercules occidentalis: Pupa hembra: A: dorsal; B: ventral; C: larva de tercer instar lateral. 
Aspectos biológicos: los datos logrados entre 2011 y 2012 sustentaron la información de ciclo de vida y medidas corporales anotados en las tablas 1 y 2. Dicha información permite inferir que el ciclo de vida y medidas corporales alcanzados por D. h. occidentalis, aparentemente, son similares a lo observado en $D$. h. lichyi, con el cual comparte una media de duración de huevo a adulto de 16 a 18 meses. Los datos tomados evidenciaron una significativa elongación del corion, que va de 1.7 a 1.8 veces; la medida corporal más discreta fue la anchura cefálica que se amplió en aproximadamente 1.8 a 1.9 veces en cada instar. El comportamiento alimenticio y de apareamiento observado en los adultos parentales también fue similar en muchos aspectos a los de D. h. lichyi. Esta subespecie ha sido mayormente registrada para la costa pacífica ecuatoriana y colombiana (Lachaume, 1994; Pardo-Locarno, 2005; Pardo-Locarno et al., 2020); la distribución ha sido recientemente documentada con detalles regionales y de rango altitudinal (Pardo-Locarno et al., 2020).

Por la gran similitud en las descripciones larvales y las razones antes expuestas, esta investigación sustenta la condición de subespecie de $D$. h. occidentalis y se aleja de la propuesta de Huang (2017) de elevarla al carácter de especie. Sobre esta última, el autor expuso dificultades para acceder a ejemplares de la región, obteniendo uno solo en el departamento de Chocó, de lo cual también se deducen dificultades en los análisis geográficos y ecológicos, tan necesarios para un mejor análisis. D. h. occidentalis ha sido quizás la subespecie mejor estudiada por autores nacionales, basados en colectas propias en el hábitat (Pardo-Locarno, 2005), algo que no oculta vacíos de monitoreo en la mayoría de la costa pacífica, ni la necesidad de más estudios específicos que aborden la distribución de la especie a lo largo de la provincia Chocó-magdalenense (Hernández-Camacho et al., 1992), en especial, en el sector nororiental de dicha provincia, en el Bajo Cauca y Bajo Magdalena (Pardo-Locarno et al., 2020).

\section{Dynastes neptunus Quenzel}

redescripción de larva de tercer

instar (Figuras 10, 11, 12, 13)

Basado en cuatro exuvias de larvas de tercer instar, fijadas en 2003 y 2004 obtenidas de la cría de inmaduros con parentales colectados en La Paz (Santander - Colombia) en abril de 2002; una prepupa y cuatro larvas de tercer instar, obtenida en La Belleza (Santander - Colombia), fijadas el 27 de diciembre 27 de 2003 y el 10 de noviembre de 2004.

La longitud de los ejemplares medida en larvas vivas fue de entre $82 \mathrm{~mm}$ y $88 \mathrm{~mm}$. La cabeza (Figura 10A) es de color pardo oscura rojiza; la anchura cefálica varió entre $14 \mathrm{~mm}$ y $16.5 \mathrm{~mm}$; de superficie rugosa, punturada, punturas en densidad de 2 $\mathrm{mm}$ a 3 por $\mathrm{mm}$, a veces coalescentes $\mathrm{y} / \mathrm{o}$ separadas por dos a tres diámetros; frente con una a dos setas frontales posteriores, una a dos setas frontales exteriores, en cada lado; una a dos pequeñas setas frontales anteriores, dos setas en el ángulo anterior de la frente; el resto de la cabeza con tres setas dorso epicraneales, tres a 20 setas 
paraocelares, basiantenales, al menos 10 a 15 setas perimetrales a cada lado; clípeo con dos setas clipeales exteriores a cada lado; una seta central clipeal a cada lado; labro asimétrico, bordes laterales con cuatro a siete setas, entre 12 y 20 setas basales y dos setas centrales; stemmata visibles, color claro-ambar. Epifaringe (Figura 1oB) con proceso haptomeral convexo, oblicuo y rodeado de setas; acanthoparia con 12 a 13 espinas robustas, ligeramente curvadas, las finales casi recta y próximas a la acroparia, la cual está conformada por cuatro a seis setas robustas; corypha reducida a tres o cuatro setas largas, gymnoparia no muy larga, con la parte media más engrosada, carente de plegmatia; chaetoparia derecha con 10 a 15 sensilas perimetrales y entre $30 \mathrm{y}$ 40 setas cortas robustas en torno al pedium; pedium largo, ensanchado; chaetoparia izquierda similar, con sensilas al borde y setas más robustas al interior; dexiotorma corta con el ápice aguzado, laeotorma larga; pternotorma pigmentada, globular; placa esclerosada triangular, aguzada; cono sensorial largo, en forma de barra, ápice redondeado; crepis poco pigmentado.

Mandíbula izquierda (Figura 11A, 11B y 11C) con dos ápices incisivos, separados por una muesca incisiva obtusa, sigue otra muesca grande y otro pequeño diente corto de ápice redondeado; en la parte media se observa un área cóncava, inerme, seguida de las coronas molares, la primera $(\mathrm{M} 1)$, grande y proyectada, las dos siguientes (M2 y M3) contiguas y bajas; acia larga y aguda, brustia pequeña; área estriduladora ventral (Figura 11B y 11C) ovalada, los 18 a 20 primeros surcos

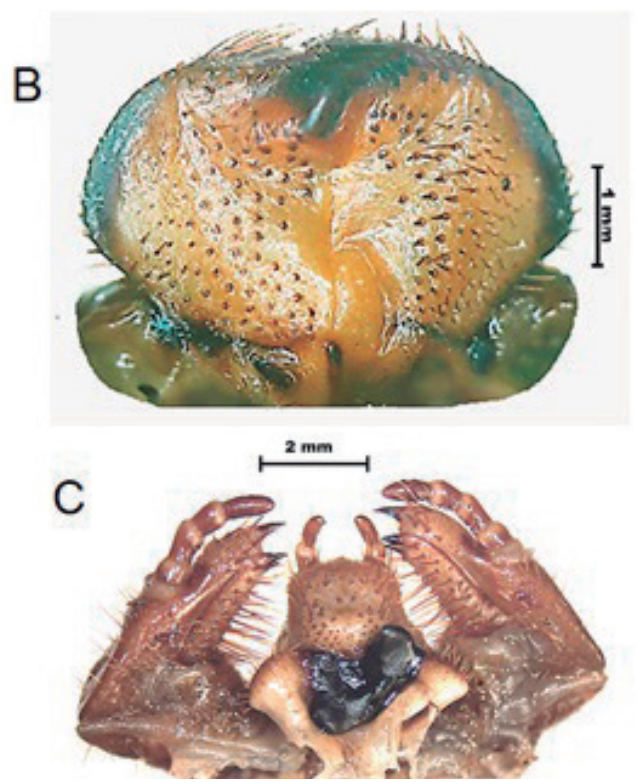

Figura 10. Dynastes neptunus. A: Cabeza; B: Epifaringe; C: Labio, máxilas. 
más amplios, los restantes 30 a 40 más delgados como líneas, enfrente de esta en su porción media se observa un pequeño mechón de setas largas; mandíbula derecha similar (Figura 11C), se diferencia en el área molar poco proyectada y contigua (M1 unido a M2 y M3); maxila galea con un uncus apical (Figura 10C), aguzado y pigmentado, lacinia con tres unci un poco más pequeños, contiguos en la base; aparato estridulador maxilar conformados por 8 a 10 dientes truncados y al final uno más grande, casi oblicuo. Labium con 30 a 36 setas en el borde basal (Figura 10C), entre 30 y 35 setas a cada lado; escleroma hipofaríngeo asimétrico, con un lado esclerosado, setoso y el otro abruptamente levantado en un diente anguloso y pigmentado, con 22 a 26 setas en el lóbulo derecho y entre 50 y 56 en el izquierdo; antenas largas y robustas (Figuras $11^{\mathrm{a}}$ y $\left.11 \mathrm{~B}\right)$, segmento apical cupuliforme con la fase dorsal dotada de 10 a 12 fosetas sensoriales (Figura 11B).
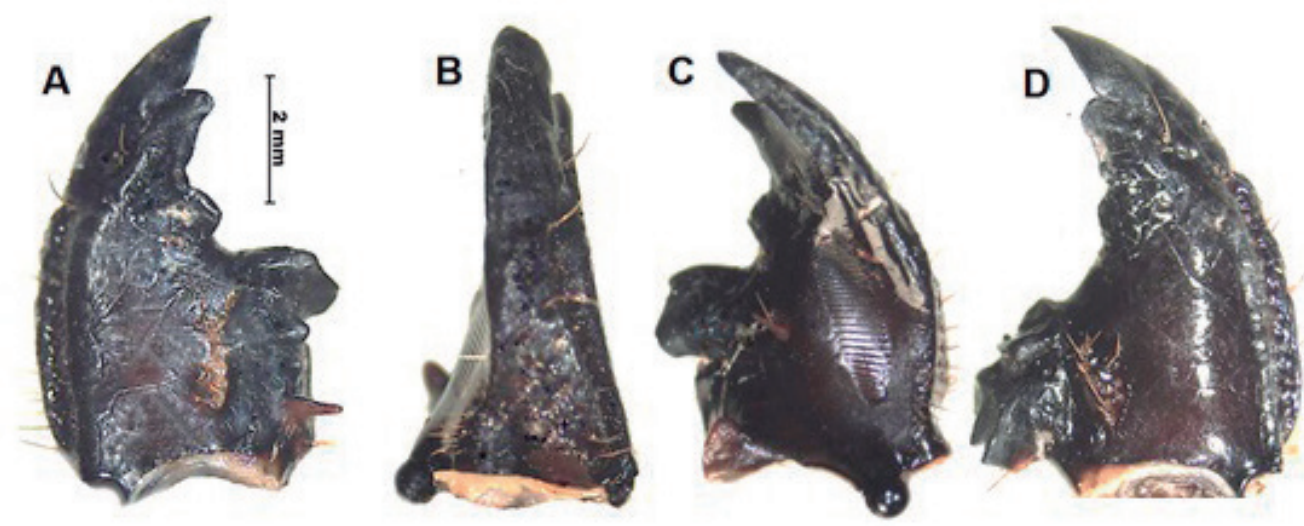

Figura 11. Dynastes neptunus. A: Mandíbula izquierda dorsal; B: Mandíbula izquierda lateral; C: Mandíbula izquierda ventral; D: Mandíbuladerecha, dorsal

El tórax presenta espiráculo torácico de entre $2.5 \mathrm{~mm}$ de alto y $1.6 \mathrm{~mm}$ aprox. de ancho (Figura 12A); placa respiratoria pardo rojiza en forma de $\mathrm{C}$ con los ápices contiguos; bulla pequeña levemente convexa (Figura 13C). Escleroma lateral largo, ambarino oscuro, con ápices agudos, disco cóncavo y pigmentado (Figura 12A); placas dorsales del protórax y mesotórax poco diferenciadas, con múltiples setas cortas espiniformes; placa dorsal del metatórax con bandas más definidas de setas cortas espiniformes y algunas setas largas. Patas (Figura 12C) grandes y robustas, densamente setosas, las pro y mesotorácicas con la uña aguzada y dos pares de setas alternas en cada punto, dos basales y dos subapicales; uña tarsal metatorácica poco aguzada, con dos setas internas y otras dos setas apicales externas (Figura 12C). 

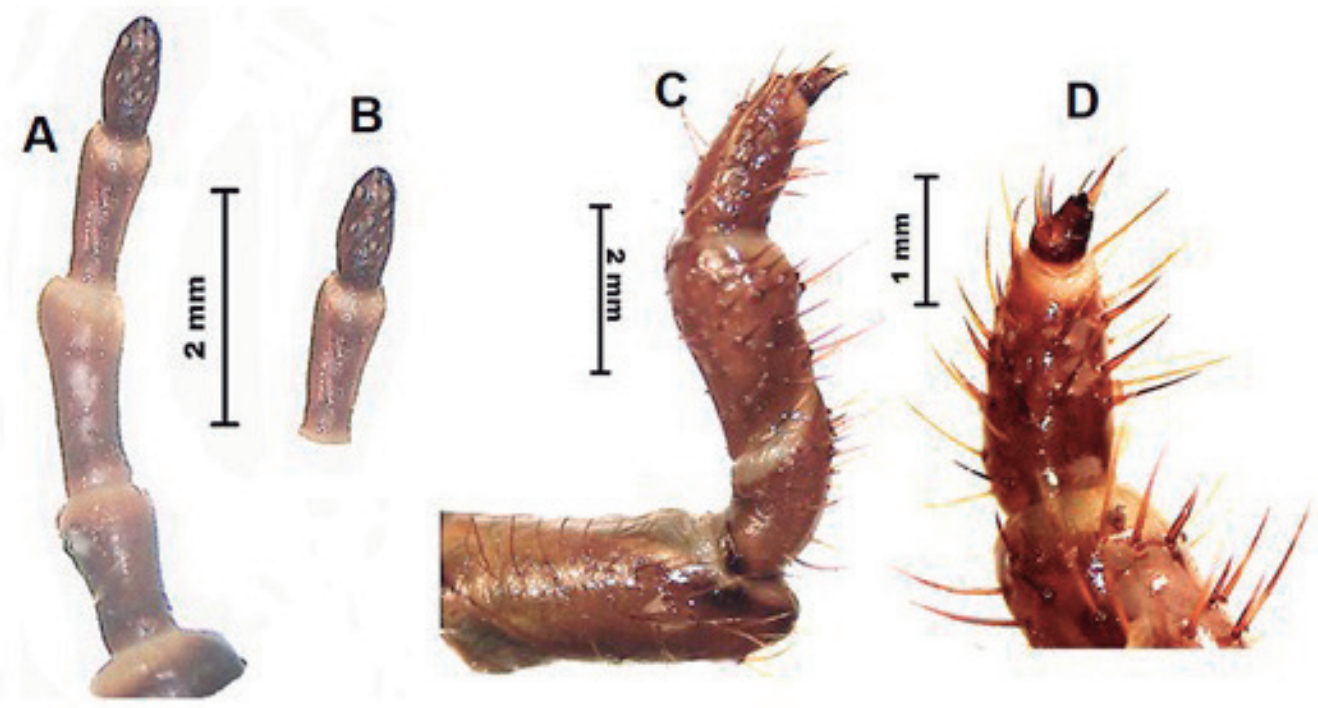

Figura 12. Dynastes neptunus. A: antena; B: último y penúltimo segmento antenal, dorsal; C: pata protorácicas; D: tarsungulo, ápice y uña.

Abdomen: espiráculos abdominales I-VIII (Figura 13A) de tamaño similar (entre $1.8 \mathrm{~mm}$ y $2.0 \mathrm{~mm}$ de alto por $1.7 \mathrm{~mm}$ a 1.8 $\mathrm{mm}$ de ancho), con placa respiratoria en forma de $\mathrm{C}$ y ápices contiguos; bulla poco proyectada; dorsa de los segmentos I-VI con cada placa (prescutum, scutum y scutellum) claramente definida, dotadas de múltiples setas cortas espiniformes y en menor grado setas largas en franjas posteriores; dorsa de los segmentos VII y VIII con placa única (Figura 13B), dotada de bandas de múltiples setas cortas espiniformes y unas pocas setas largas en el borde posterior; dorsa IX similar a VIII; venter I-IX dotado de franjas de entre 20 y 30 setas cortas espiniformes y cinco setas largas; ráster sin palidia (Figura 13D), campus con entre 10 y 12 setas cortas espiniformes y entre 8 y 10 setas largas entremezcladas; tege triangular dotado de entre 28 y 32 setas, entre 16 y 18 setas cortas espiniformes y de 8 a 10 setas largas; labio anal inferior con poco más de 100 setas cortas espiniformes y una cifra similar de setas largas casi alineadas al borde del labio anal; barbula densamente setosa, setas largas; abertura anal larga, poco curvada.

Dynastes neptunus Quenzel. Pupa: descripción de macho y redescripción de hembra. (Figuras 14, 15 y 16).

En la descripción de inmaduros de D. neptunus (Onore \& Morón, 2004), se describió detalladamente el caso de la pupa de hembra, dejando con pocos elementos la del macho, la cual tenía problemas de preservación; igualmente dicho trabajo ilustró pupas macho y hembra con una vista somera 

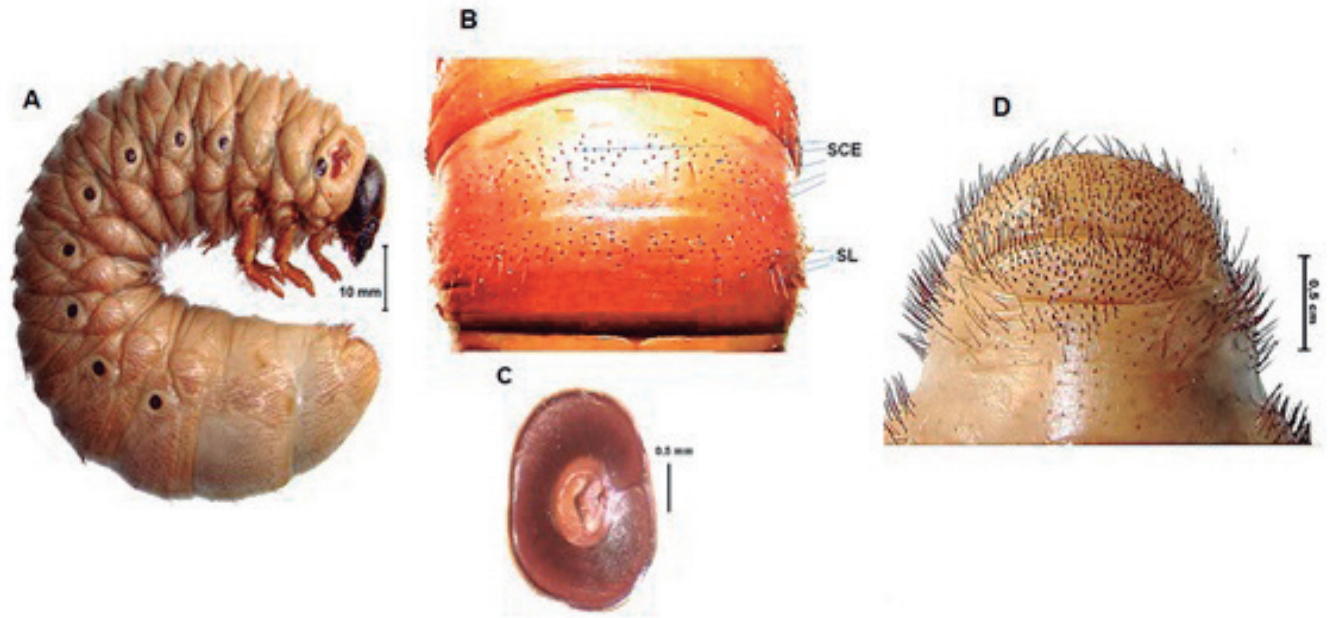

Figura 13. Dynastes neptunus. A: larva de tercer instar, lateral; B: dorsal segmento abdominal VII; C: estigma respiratorio torácico; D: abdomen, ráster (Abreviaturas: SCE: setas cortas espeinifirmes. SL: setas largas.

y algo oscura de los ejemplares; esta nota se propuso subsanar este vacío describiendo e ilustrando ambos casos.

La descripción se basó en cuatro pupas (tres masculinas y una femenina) fijadas en 2003 y 2004, obtenidas de larvas criadas a partir de adultos colectados en La Paz (Santander - Colombia), a una altura de 1.500 a $1.600 \mathrm{msnm}$, en abril de 2002; dos exuvias pupales fijadas en abril de 2005 obtenidas de la cría de inmaduros con parentales colectados en La Paz, en abril de 2002; una exuvia pupal fijadas en 2007 obtenida en diciembre de 2004 en La Belleza (Santander - Colombia).

Descripción del macho (Figura 14): longitud corporal de entre $78 \mathrm{~mm}$ y $79 \mathrm{~mm}$; exarata, cuerpo robusto, ovalado, color amarillo oscuro, con pequeñas manchas o lunares negros dispersos en todo el cuerpo; cabeza ubicada en posición ventral al protórax (Figura 14B y 14C), región frontal abultada, proyectada, con un cuerno corto (macho minor); la región anterior de la cabeza bosqueja los ápices de estructuras bucales y sensoriales (Figura 16C), clípeo transverso, tumido, bilobulado, con una leve muestra central anterior en forma de "Y", labro ubicado centralmente bajo el clípeo (Figura 16C); ápices mandibulares $\mathrm{y}$ antenales visibles, palpos maxilares y labiales con anillos visibles; ojos casi ocultos; labium constreñido por los palpos; Tórax, pronotum transverso, dotado de cuerno (Figura 16C); borde anterior sinuado, con proyecciones laterales que rodean la cabeza, borde posterior levemente bisinuado con los ángulos posteriores bastante redondeados; 
mesotórax dorsalmente visible, borde anterior recto y el posterior centralmente proyectado en forma obtusa; sutura ecdisial longitudinal visible; proceso proesternal visible y proyectado en forma de disco (Figura 14A); mesosternum oculto parcialmente bajo las podo y pterotecae (Figura 14A); metanoto grande, anteriormente biescotado, borde posterior casi recto, levemente sinuado y surco ecdisial longitudinal visible, en la parte anterior expone un bosquejo triangular; pterotecae mesotorácicas dorsal y lateralmente visibles y muy proyectadas, las pterotecae metatorácicas parcialmente ocultas, visibles al ápice (Figuras 15B y ${ }_{15} \mathrm{C}$ ); podotecae bastante visibles, largas, las protorácicas dirigidas hacia la región esternal, las meso y metatorácias hacia los lados y atrás, dejando ver a los extremos los anillos tarsales (Figura 14C). Abdomen con nueve segmentos basalmente más anchos (Figura 14); espiráculo I presente, oculto bajo el borde posterior torácico (visible en exuvias pupales); espiráculos II, III y IV, visibles dorsalmente (Figuras $14^{\mathbf{a}} \mathbf{y}$ 14B), en todos los casos con los peritremas pigmentados, ovalados, casi tres veces más largo que ancho; V, VI y VII ocluidos, vestigiales, carentes de peritrema esclerosado, VIII no visible; región tergal de los segmentos I-VII con seis pares de órganos dioneiformes, bien definidos y medianamente pigmentados (Figuras $15^{\mathbf{a}} \mathbf{y}$ 15B); órgano dioneiforme VI no pareado (Figura 14A), porción proximal no definida, porción posterior bien formada; ese mismo tergito presenta, bosquejado en el borde posterior, el rastro de una valva de órgano dioneiforme muy vestigial (Figura
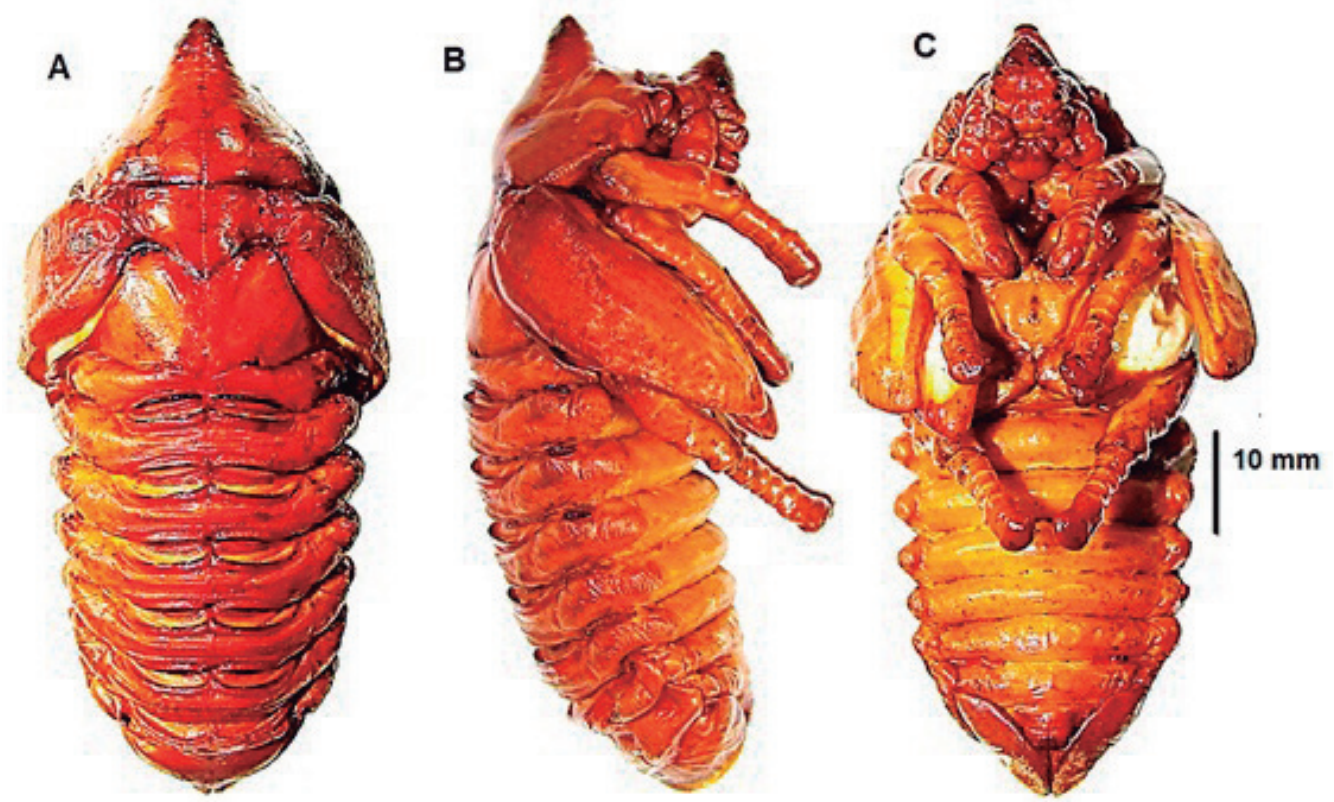

Figura 14. Dynastes neptunus. Pupa macho: A: dorsal; B: ventral; C: lateral. 
16D a); área tergal del segmento VIII con dos profundas oquedades en forma de riñón (Figura 16D b); segmento IX aguzado, triangular, apicalmente bifurcado, con una densa cobertura setosa dorada; esternito I oculto bajo la podotecae; esternitos II, III, IV, V, VI y VII muy cortos en forma de franjas convexas, con ápices laterales en forma de muñones; esternitos VIII y IX aguzados, en la región ventral dejan ver el ámpula genital masculina, con forma de cilindro, en dos piezas contiguas, la apical redondeada.

Hembra: redescripción (Figura 15), longitud de entre $66 \mathrm{~mm}$ y $67 \mathrm{~mm}$, anchura máxima del tórax entre $29 \mathrm{~mm}$ y $30 \mathrm{~mm}$; cuerpo muy similar al del macho, del cual difiere por algunos pocos rasgos genéricos. Cuerpo robusto, ovalado, color amarillo oscuro; cabeza en posición ventral al protórax (Figuras 15B y 15C); frente abultada con sendas oquedades a cada lado (Figura 15D); estructuras bucales y sensoriales visibles similares a las del macho. Tórax, pronotum convexo, disco inerme, con una corta proyección anterior en forma de tubérculo (Figura 15A); abdomen con el espiráculo I oculto, los espiráculos II, III y IV visibles, con peritrema pigmentado (Figura 15A); los siguientes V, VI y VII ocluidos, vestigiales (Figuras $15^{\mathbf{a}}$ y $15 \mathrm{~B}$ ); órganos dioneiformes presentes en los segmentos I a VII, en I a VI pareados (Figura 15A), en VII no pareado (Figura 15B); ámpula genital levemente convexa, con bosquejo de genitales, ápice abdominal que culmina en una orqueta pubescente dotada de setas finas doradas.

Aspectos biológicos: Sobre esta especie y su ciclo de vida existen muchas imágenes y datos, pero ningún trabajo formal fue observado sobre su ciclo de vida; Onore \& Morón (2004) realizaron varios aportes biológicos y ecológicos, como la definición
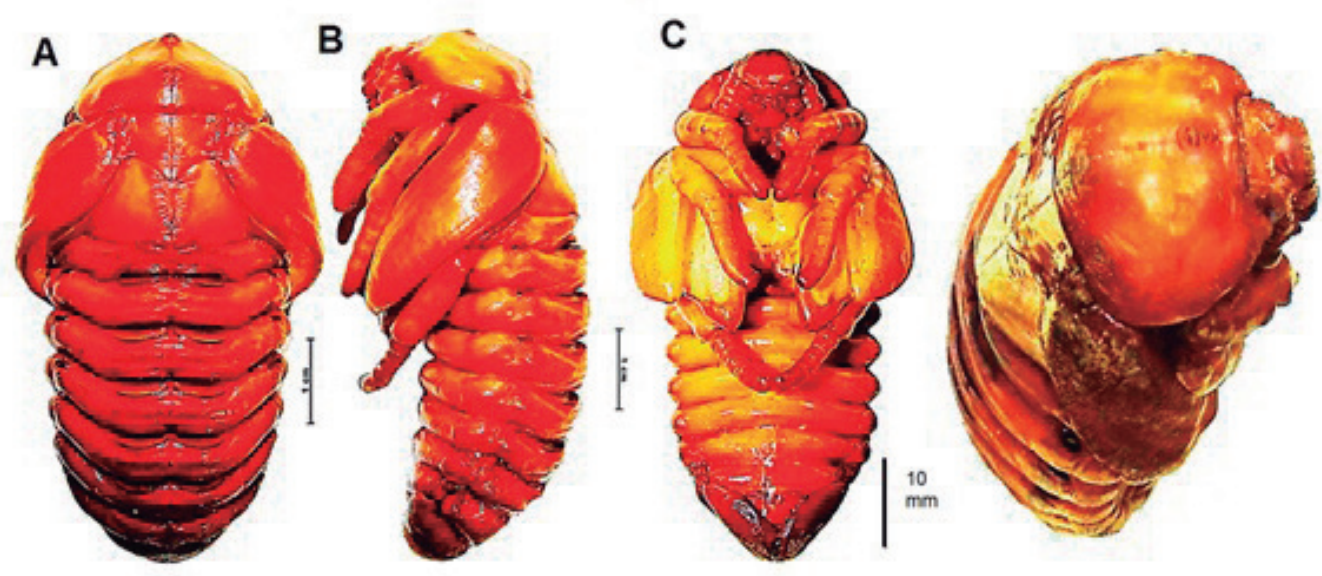

Figura 15. Dynastes neptunus. Pupa hembra: A: dorsal; B: lateral; C: ventral; D: protórax, dorso-lateral. 
de su hábitat, sustrato de las larvas y adultos, comentarios sobre las cámaras pupales. Tras lidiar con obstáculos logísticos, locativos y legislativos, se logró obtener los datos anotados en las tablas 1 y 2, que resumen dos ensayos realizados desde 1998 hasta 2004; a reserva de examinar con más detalle y en un espacio mayor dichos logros (PardoLocarno, in lit), por ahora podría exponerse que el ciclo de vida varió de 1.4 a 2.5 años, evidenciándose que las medias del ciclo de vida de esta especie fueron ligeramente más larga que la de $D$. hercules, observándose varios casos que demoraron tres años o más para llegar al estado adulto, con algunos casos que sobrepasaron esa cifra y que por ahora, se excluyeron de la tabla, mientras se logra una mejor interpretación de los resultados. Los estados de crecimiento evidenciaron datos más discretos en cuanto a la anchura cefálica, variando en proporciones aproximadas de 1.7 a 1.9 entre el instar I y el III. De manera similar, las excretas larvales variaron de manera discreta desde $4 \mathrm{~mm}$ de largo hasta los 2.2 $\mathrm{mm}$ de ancho en instar I, $8 \mathrm{~mm}$ a $9 \mathrm{~mm}$ de largo y entre $4.5 \mathrm{~mm}$ y $5 \mathrm{~mm}$ de ancho en instar II y entre $14 \mathrm{~mm}$ y $15 \mathrm{~mm}$ de largo y $5 \mathrm{~mm}$ de ancho en las de último instar (Figura 16A). A nivel de huevo se observó la elongación del corion, que va de 1.5 a 1.6 veces (Figura 16B); las medias de anchura y longitud corporal de larvas de II y III instar, se correspondieron con ejemplares de talla pequeña y mediana (machos entre $57 \mathrm{~mm}$ y $62 \mathrm{~mm}$ y hembras de entre $52 \mathrm{~mm}$ y $54 \mathrm{~mm}$ ), inferiores a las de los parentales.

Aunque varios aspectos podrían ser argumentados, por ejemplo, problemas dietarios (Karino et al., 2004), surgen inquietudes en torno a la temperatura media, algo que se correspondería con el hecho de que los parentales se colectaron a 1900 msnm, o sea, la típica distribución altoandina de la especie (Pardo-Locarno et al., 2020). Ello no descartaría a los requerimientos alimenticios, como las características de la madera descompuesta o el sustrato derivado de árboles específicos (Onore \& Morón, 2004). En la búsqueda de respuestas concretas y con la intención adicional de lograr ciclos más cortos y de cohortes más grandes, se recomienda optar por cambios sustanciales, como es la cría in situ, y explorar su articulación a procesos comunitarios en mercados verdes, que pudieran revertir los efectos negativos del comercio ilegal (Amat et al., 2003). D. neptunus ha presentado la distribución tropandina más extendida del género en Colombia, evidenciando datos de colecta en las cuencas de los ríos Cauca y Magdalena y en las tres cordilleras colombianas, en altitudes que varían de 1.500 msnm a 2.500 msnm (Pardo-Locarno, 2005; Pardo-Locarno et al., 2020); aspectos más detallados sobre la distribución departamental y altitudinal de esta especie en Colombia han sido compilados por el primer autor (Pardo-Locarno, in litt).

\section{Clave para larvas de tercer instar de las} especies de Dynastes (Basado en Ritcher, 1966; Morón, 1987; Onore \& Morón, 2004; Villamarín-Cortes et al., 2016)

1. Cada uña tarsal con cuatro setas apicales, dos setas internas, contiguas, en posición basal y otras dos setas externas, 
contiguas, en posición subapical (Figuras 12C y 12D). Acanthoparia con nueve a 13 setas robustas curvadas. Región dorsal del último segmento antenal con cinco a 10 fosetas sensoriales (Figuras $12 \mathrm{~A} \mathrm{y}$ 12B). Venezuela, Colombia hasta Perú . Dynastes (Theogenes) neptunus Quenzel.

$1^{\prime}$ Cada uña tarsal con solo dos setas, una seta interna, en posición basal y otra seta externa en posición apical; demás caracteres diferentes 2

2. Acanthoparia con cinco a seis setas robustas curvadas. Teges con 15 a 16 setas cortas espiniformes. Chaetoparia derecha de la epifaringe con seis a ocho setas cortas; dos a tres setas dorsoepicraneales. México, Honduras Dynastes hyllus Chevrolat.

2' Acanthoparia con 10 a 16 setas robustas curvadas. Setas frontales anteriores ausentes; otros carateres diferentes 3

3. Acanthoparia con 14 a 16 setas robustas curvadas, contigua a la acroparia; dos setas anteriores frontales a cada lado 4

3' Acanthoparia con 10 a 12 setas robustas curvadas, demás caracteres diferentes

4. Teges con 10 a 14 setas hamatae; chaetoparia derecha de la epifaringe con pocas o ninguna sensila. USA Dynastes granti Horn.

4' Teges con $16-35$ setas hamatae; chaetoparia derecha de la epifaringe con numerosas sensilas. USA

Dynastes tityus (L.)

4. Cabeza con cuatro a cinco setas dorsoepicraneales; región dorsal del último segmento antenal con 10 a 12 fosetas sensoriales. Colombia (cuenca del Río Magdalena y Cordillera Oriental), Venezuela, Ecuador, etc

Dynastes hercules lichyi Lachaume.

4' Cabeza con dos a tres setas dorsoepicraneales; región dorsal del último segmento antenal con seis a ocho fosetas sensoriales. Colombia (costa pacífica), Ecuador (occidente)

Dynastes hercules occidentalis Lachaume.

\section{Key to Species of Dynastes Larvae}

(Modified from Richter, 1966; Morón, 1987; Onore \& Morón, 2004;

Villamarín-Cortes et al., 2016)

1. Each tarsal claw with two internal, nearly basal setae, and two external,preapical setae (Figures 12C y 12D). Dorsal surface of last antennal segment with five to 10 sensory spots. Colombia, Ecuador, Perú, Venezuela

Dynastes (Theogenes) neptunus Quenzel.

$1^{\prime}$ Each tarsal claw with one internal, nearly basal setae, and one external, preapical setae .. 2

2. Acanthoparia with five to six spine-like, short setae. Teges with 15-16 short setae. Right chaetoparia of epipharynx with six to eight sensilla. México, Honduras .... Dynastes hyllus Chevrolat. 
2' Acanthoparia with 10-12 spine-like, short setae. Anterior frontal setae absent .. 3

3. Acanthoparia with 13-14 spine-like, short setae. Two anterior frontal setae ...... 4

3' Acanthoparia with 10-12 spine-like, short setae, other different characters ....... 5

4. Teges with 10-14 short, flattened tapering setae. Right chaetoparia of epipharynx with 2-4 sensilla or without sensilla. Head capsule with six to eigh dorsoepicranial setae on each side. USA

Dynastes granti Horn.

4' Teges with 15-35 short, flattened tapering setae. Right chaetoparia of epipharynx with 12 to 20 sensilla. USA Dynastes tityus (Linné).

5. Head capsule with four to five dorsoepicranial setae. Dorsal surface of last antennal segment with 10-12 sensory spots. Magdalena River, eastern mountain range of Colombia, Venezuela, etc. ........ Dynastes hercules lichyi Lachaume.

5 Head capsule with two or three dorsoepicranial setae. Dorsal Surface of last antennal segment with six to eight sensory spots. Pacific coast of Colombia and Ecuador Dynastes hercules occidentalis Lachaume.

\section{Clave para pupas de las especies colombianas de Dynastes Kirby}

1. Borde posterior del área tergal VII con el rastro de una valva de órgano dioneiforme muy vestigial (Figura 16E a). Área tergal del segmento VIII con dos profundos hoyuelos en forma de riñón (Figura 16E b) ...... Dynastes (Theogenes) neptunus Quenzel.

$1^{\prime}$ Borde posterior del área tergal VII con leve sinuado, sin rastro de valva de órgano dioneiforme vestigial (Figura 16D a). Área tergal del segmento VIII con dos oquedades largas y someras (Figura 16D b) Dynastes hercules (L). 2

2. Zona media dorsal de las áreas tergales IV, V, VI y VII con una banda grisácea transversa (Figura $1 \mathrm{~B}, 1 \mathrm{C}$ ), nítidamente visible, con amplitud cercana al ancho de los órganos dioneiformes, cuando presentes D. h. lichyi Lachaume.

$2^{\prime}$ Zona media dorsal de las áreas tergales IV, V, VI y VII carentes de banda grisácea transversa (Figura 8A), si presente, poco definida, poco visible, del mismo color del área tergal D. h. occidentalis Lachaume.

\section{Key to Colombian Species of Dynastes pupa}

1. Posterior border of tergal area VII with the trace of a very vestigial dioneiform organ valve (Figure $16 \mathrm{E}$ a). Tergal area of segment VIII with two kidney-shaped, deep impressions Dynastes neptunus Quenzel.

$1^{\prime}$ Posterior border of tergal area VII without trace of vestigial dioneiform organ valve; Tergal area of segment VIII 

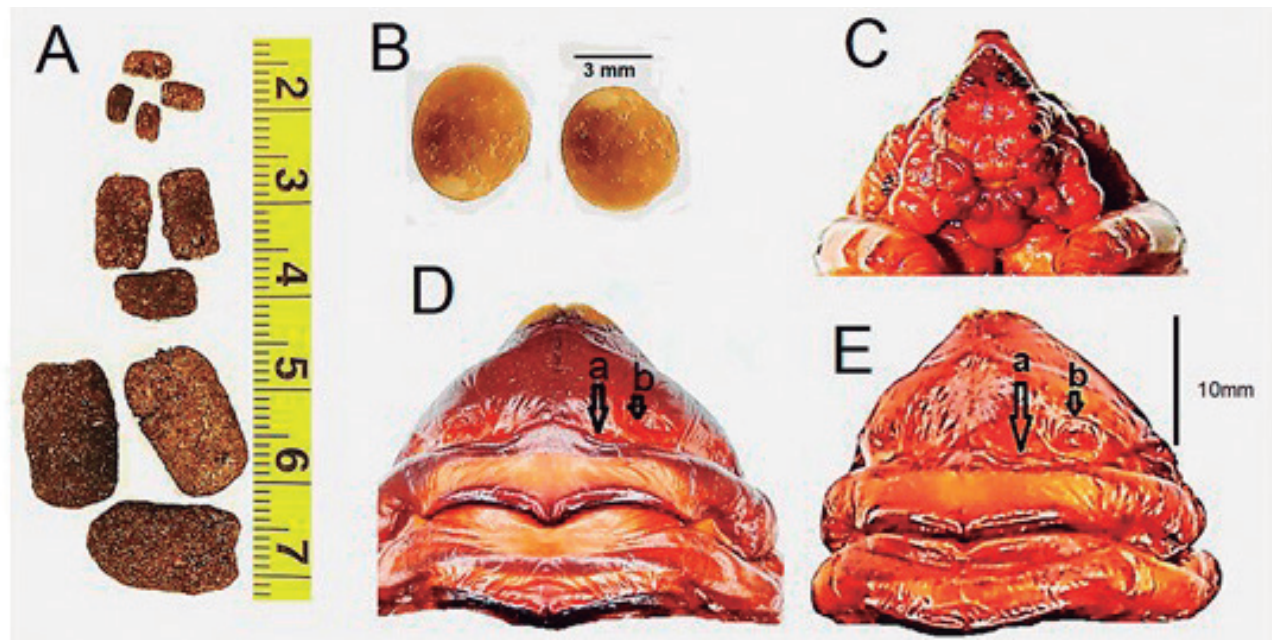

Figura 16. Dynastes neptunus. A: excretas de todos los estadios; B: huevos elongados;

C: pupa macho, cabeza vista ventral; D: pupa Dynastes hercules lichyi, borde posterior de la dorsa del VII y VIII segmento abdominal; pupa Dynastes neptunus, borde posterior de la dorsa del VII y VIII segmento abdominal (Abreviaturas: a: borde posterior de la dorsa VII; b: lateral de la dorsal del segmento VIII).

with two long and shallow cavities Dynastes hercules (L)

2. Middorsal area of tergal areas IV, V, VI and VII with a transverse gray band, clearly visible (Figure $1 \mathrm{~B}, 1 \mathrm{C}$ ), with width close to the width of the dioneiform organs D. h. lichyi Lachaume.

$2^{\prime}$ Middorsal area of tergal areas IV, V, VI and VII lacking transverse grayish band, if present, poorly defined, not very visible, of the same color as the tergal area D. h. occidentalis Lachaume.

\section{Conclusiones}

Los estudios de Richter (1966) y de Onore \& Morón (2004) dejaron ver las dificultades de describir larvas longevas de Dynastes, lo que propicia vacíos o ambigüedad descriptiva. Esta investigación confirmó el desgaste de integumento, del aparato bucal, pérdida de múltiples setas cefálicas y de la uña tarsungular. Este desgaste resulta más evidente en piezas del tejido exuvial, algo que se subsanó por la afortunada disponibilidad de ejemplares bien dotados. La quetotaxia de los apéndices (tarsúngulo), de los teges, de la acanthoparia y del dorso epicráneo se consideran, por ahora, focales en este género. La acanthoparia de Dynastes hercules se observa más dotada de setas (entre 12 y 14), algo en lo que coincide con la figura 13 de Villamarín-Cortez et al (2016). En lo referente a la descripción de larvas de tercer instar de D. neptunus, se observó menor variabilidad en los datos 
de la quetotaxia cefálica, respecto a los registrados por el Maestro Morón (Onore \& Morón, 2004), algo que podría atribuirse al estado de conservación de los ejemplares disponibles para la descripción.

La morfología de inmaduros (larvas) de Dynastes podría aportar mucho a la discusión de la taxonomía del género. De acuerdo con este trabajo y los estudios consultados (Morón, 1987; Onore y Morón, 2004; Richter, 1966) la morfología larval del género evidencia una gran similitud, siendo relativamente muy afines D. hyllus Chevrolat, D. granti Horn, D. tityus (Linne) y D. hercules; esta cercanía fue mayor en el caso de las larvas de las dos subespecies de Dynastes hercules aquí estudiadas, algo que por ahora no apoya o descarta erigir especies (Huang, 2017). En este escenario, Dynastes neptunus Quenzel fue el caso que evidenció mayor divergencia.

Los estados pupales constituyen una fase muy conservadora de la morfología de los Melolonthidae (Morón, 1993); en ese sentido, las pupas de Dynastes conocidas D. hyllus Chevrolat y D. neptunus Quenzel (Morón, 1987; Onore \& Morón, 2004) y las aquí descritas (dos subespecies de D. hercules) evidenciaron una notable similitud morfológica, escenario en el cual D. neptunus, nuevamente, mostró divergencia en cuanto a los caracteres dorsales de los segmentos VII y VIII.

El género Dynastes MacLeay, 1819, incluye los subgéneros Dynastes y Theogenes Burmeister, 1847, este último con dos especies exclusivamente andinas y suraméricanas, distinguibles por el dorso negro y el pretarso engrosado (Endrodi, 1976; Huang, 2017; Hwang, 2011), estas son D. neptunus Quenzel y D. satanas Moser. El subgénero Theogenes Burmeister ha sido desestimado a lo largo del tiempo (Endrodi, 1976; Hwang, 2011), sin embargo ha sido afirmado en esta publicación, pues se ha evidenciado clara divergencia en cuanto a la morfología larval y la de la pupa, algo que serviría para consolidar su condición de subgénero y, quizás, de hipótesis para restablecerlo como género válido, dejando un manto de inmensa inquietud en torno a la morfología larval de su símil $D$. satanas de Yungas Bolivia (Endrodi, 1976; Hwang, 2011)

\section{Agradecimientos}

Múltiples circunstancias se conjugaron en la iniciativa personal de sacar adelante esta investigación de tantos años, por ello doy gracias a la vida y a un ser superior, porque entre tantos limitantes y largo tiempo de trabajo fue posible terminar este carísimo informe. El primer autor agradece a sus compañeros de investigación en especial al ingeniero Andrés Felipe Serna por el inmenso apoyo de laboratorio durante años y a Libardo Suárez Fonseca (Corponor), fiel colega y amigo, por facilitar el complejo trámite para la visita, colecta y transporte de los ejemplares para estudio científico. Durante 1998 y 1999 al inicio de los ensayos se contó con el apoyo del señor Javier Sinchi. Elena de la Concepción GómezDuque y Harold Villota apoyaron la fase de laboratorio entre 2008 y 2010; Velia Carolina Pardo apoyó la fase de laboratorio 
(2010- 2012). El primer autor agradece el acompañamiento en campo y la donación de ejemplares por parte de la empresa Zoonatura (familia Urbina) y la familia Vargas (Otanche y La Belleza, Santander - Colombia); en el Valle del Cauca a José Murillo y Amparo Bubú (Consejo Comunitario del Alto y Medio Dagua-CCAMDA) por el apoyo en campo y cría en 2011 y 2012. Se agradece el apoyo institucional de Julián Rentería (CVC), Enrique Murgueitio y Gunnar Mordhorst (CIPAV); así mismo del IIAP (1999- 2003; 2009), CVC (proyectos 1995-96, 2005; 2011-2012), Corpoamazonia (Alex Mejía, PhD. e ingeniera Ligia Stella Peñafiel) y a la Universidad del Pacífico (2014-2020). Agradecimientos por la bibliografía compartida por Fernando Vaz de Mello y Miguel Ángel Morón, igualmente a Paul Schoolmeester.

\section{Referencias}

Amat, G., Gasca, H., y Amat, E. 2003. Guía para la cría de Escarabajos. Fundación Natura - Universidad Nacional de Colombia. Bogotá, Colombia. 8 o p.

Ardila, N.Y y Sánchez, P. 2017. Relación entre las características del hábitat y la producción de individuos adultos de Dynastes hercules en relictos de bosque con diferente esfuerzo de caza. Acta Biológica Colombiana, 11(1), 141-142. Disponible en: https:// revistas.unal.edu.co/index.php/actabiol/article/view/27506

Arrow, G. 1951. Horned Beetles: A study of Fantastic in Nature. W. Junk, Publishers, The Hague. Netherland. 18o pp.

Ballesteros-Meza, L.F. y Amat-García, G. 2006. Caracterización de hábitat y abundancia local de Dynastes hercules (Coleoptera: Melolonthidae: Dynastinae) en bosques subandinos del departamento de Santander, Colombia. Acta Biológica Colombiana, 11(1), 170. Disponible en: https://revistas.unal.edu. co/index.php/actabiol/article/view/27581

Blackwelder, R.E. 1944. Checklist of the coleopterous insects of Mexico, Central America, the West Indies, and South America, 
pt. 2. Bulletin of the United States National Museum. i-iii, 189341. Disponible en: https://doi.org/10.5479/si.03629236.185.2

Costa, C., Vanin, S.A. y Casari-Chen, S. 1988. Larvas de coleoptera do Brasil. São Paulo: Museu de Zoologia. Disponible en: https://doi.org/10.5962/bhl.title.100233

Dechambre, R. P., 1980. Le genre Dynastes (Col. Scarabaeoidea Dynastidae). Bulletin Sciences Nat, 27: 5-10.

Dupuis, F. y Dubois, D. 1997. Etude des facteurs contrôlant la croissance larvaire de Dynastes hercules lichyi (Coleoptera Dynastidae). Bulletin de la Société entomologique de France, 102 (4): 355-362. Disponible en: https://doi.org/10.3406/ bsef.1997.17356

Eberhard, W.G. 1977. "Fighting Behavior of Male Golofa Porteri Beetles (Scarabeidae: Dynastinae)", Psyche: A fournal of Entomology, 84 (3-4): 292-298. Disponible en: https:/doi. org/10.1155/1977/19030

Eberhard, W. G. 1980. Horned beetles. Scientific American, 242(3), 166-183.

Emlen, D.J., Hunt, J., Simmons, L.W. 2005. Evolution of sexual dimorphism and male dimorphism in the expression of beetle horns: phylogenetic evidence for modularity, evolutionary lability, and constraint. Am Nat. 166: S42-S68. Disponible en: https://doi.org/10.1086/444599

Endrödi, S. 1985. The Dynastinae of the World. Akadémiai Kiadó, Budapest. 800 pp.

Endrödi, S. 1976 Monographie de Dynastinae 6. Tribus: Dynastini Acta Zoologica Academiae Scientiarum Hungaricae, 22(3-4): 217-269.

Figueroa Potes, A., 1977. Insectos y Acarinos de Colombia. Facultad de Ciencias Agropecuarias, Universidad Nacional de Colombia, Palmira: 685 pp. 
Gasca-Álvarez, H.J. 2011. Beetle breeding and its applicability in conservation. Scarabs 59: 1-5.

Gasca-Álvarez, H.J., y Amat-García G. 2010. Synopsis and key to the genera of Dynastinae (Coleoptera, Scarabaeoidea, Scarabaeidae) of Colombia. Current advances in Scarabaeoidea research. ZooKeys 34: 153-192. Disponible en: https://doi. org/10.3897/ zookeys.34.309

Gasca-Álvarez, H.J., López-García, M. y Brett Ratcliffe. 2018. Dynastinae de Colombia (Coleoptera: Scarabaeidae): estado actual de conocimiento. En Escarabajos del Neotrópico. Cuautemos Deloya \& Héctor Jaime Gasca-Álvarez Coordinadores. P 98-128. México. ISBN: 978-607-7552-26-0.

Gruner, L. y Chalumeau, F. 1977. Biologie et élevage de Dynastes h. hercules in Guadeloupe (Coleoptera Dynastinae). Annales de la Société Entomologique de France, 13: 613-624.

Hernández-Camacho, J. y Sánchez Páez, H., 1992. Biomas Terrestres de Colombia. En: G. Halffter (compilador). La diversidad biológica de Iberoamérica I. Instituto de Ecología. A. C. Xalapa, Veracruz, México, pp. 153-173.

Hernández-Camacho, J., Hurtado Guerra, A., Ortiz Quijano, R. y Walschburger, T. 1992. Unidades Biogeográficas de Colombia. En: G. Halffter (compilador). La diversidad biológica de Iberoamérica. Instituto de Ecología, A. C. Xalapa, Veracruz, México, pp. 105-151.

Huang, J.P. 2017. The Hercules beetles (subgenus Dynastes, genus Dynastes, Dynastidae): a revisionary study based on the integration of molecular, morphological, ecological, and geographic analyses. Miscellaneous Publications Museum of Zoology, University of Michigan, No. 206 Ann Arbor. P 1-27. ISSN $0076-8405$.

Hwang, S-M-R. 2011. The Dynastini of the World. Nature and Ecology (Academic Series), Volume 4. Seoul, South Korea. 368 pp. 
Karino, K., Seki, N. y Chiba, M. 2004. Larval nutritional environment determines adult size in Japanese horned beetles Allomyrina dichotoma (author's version). Ecological Research, 19(6): 663-668. Disponible en: https://doi. org/10.1111/j.1440-1703.2004.00681.x

Lachaume, G., 1985. Dynastini 1. The Beetles of the World. Sciences Nat., Venette, 5: 85 pp.

María, Hno. Apolinar (Nicolas Seiler). 1944. Vocabulario de Términos Vulgares en Historia Natural Colombiana. Revista de la Academia Colombiana de Ciencias Exactas, Físicas y Naturales. 6 (21) :16-31, p. 18.

María, Hno Apolinar (Nicolas Seiler), 1945. Vocabulario de Términos Vulgares en Historia Natural Colombiana. Revista de la Academia Colombiana de Ciencias Exactas, Físicas y Naturales. 6 (22-23): 172-204, 2 pls: 173 y 201.

Morón, M. A. 1984. Escarabajos 200 millones de años de evolución. Instituto de Ecología. México D.F. 132 pp.

Morón, M. A., 1987. Los estados inmaduros de Dynastes hyllus Chevrolat (Coleoptera Melolonthidae Dynastinae); con observaciones sobre su biología y el crecimiento alométrico del imago. Folia Entomológica Mexicana, 27: 33-74.

Morón, M. A. 1993. Observaciones comparativas sobre la morfología pupal de los Coleoptera Melolonthidae neotropicales. Giornale italiano di Entomologia, 6(33): 249-255.

Morón, M. A. 2009. El género Dynastes MacLeay, 1819 en la Zona de Transición Mexicana (Coleoptera: Melolonthidae: Dynastinae). Boletín Sociedad Entomológica Aragonesa 45: 23-38.

Morón, M. A. \& Pardo Locarno, L. C., 1994. Larvae and Pupae of Golofa Hope (Coleoptera: Melolonthidae, Dynastinae) from Colombia. The Coleopterists' Bulletin, 48 (4): 390-399. 
Onore, G. y Morón, M. 2004. Dynastes neptunus Quenzel (Coleoptera: Scarabaeidae: Dynastinae); descripción de la larva del tercer estadio y pupa, con notas sobre su biología. Coleopterists Bulletin, 58, $103-110$.

Pardo Locarno, L. C., 1993. Estudio Preliminar de las Especies de Melolonthidae del Valle del Cauca, Colombia, con énfasis en la Cuenca Calima San Juan (Valle-Chocó). en: Diversidad y Manejo de Plagas Subterráneas. Publicación Especial de la Sociedad Mexicana de Entomología, pp 83-90.

Pardo-Locarno, L. C. 1997. Estudios Básicos para la Zoocría de Insectos Coleópteros con fines Comerciales. Documento interno GVC. 57pp. Centro de documentación CVC-Cali, Colombia.

Pardo Locarno, L. C. 20o6. Estudio de caso: Zoocría de Coleopteros en el Pacífico del Valle del Cauca. En: Enrique Murgueitio 2006. Uso Sostenible de la biodiversidad en ecosistemas y hábitats transformados. Chaves, M. E. y Santamaría, M. (Eds). 2006. Informe sobre el avance en el conocimiento y la información de la biodiversidad 1998-2004. Instituto de Investigaciones de Recursos Biológicos Alexander von Humboldt. Bogotá D. C. pp 400-401.

Pardo Locarno, L. C. 2011. Proyecto Aunar esfuerzos técnicos y económicos para mitigar el impacto sobre los recursos naturales y el medio ambiente a través de la implementación de sistemas productivos sostenibles en Sabaletas, Cordoba y Bajo Dagua, Municipio de Buenaventura, Convenio. 218-2010. CVC-AAFP. Informe técnico final (Documento interno CVC). Pp 50.

Pardo Locarno, L. C. 2005. Sinopsis preliminar de los Dynastini (Coleoptera:Scarabaeoidea) del Chocó Biogeográfico, Colombia. Boletín Científico Museo Historia Natural Universidad de Caldas. 9, 206-221. 
Pardo-Locarno, L. C., Villalobos-Moreno, A. y Franco, M. P. 2020. Escarabajos Dynastini Macleay, 1819 (Coleoptera: Scarabaeoidea: Melolonthidae) de Colombia. En: VII Curso Escarabajos de Colombia (Coleoptera: Scarabaeoidea) taxonomía, biología y ecología. LC Pardo-Locarno, MC Gallego-Ropero \& J Montoya-Lerma Editores. Popayán, Cauca. Pp: 101-116. ISBN: 978-958-49-1590-0.

Ratcliffe, B. C. 2003. The dynastine scarab beetles of Costa Rica and Panama (Coleoptera: Scarabaeidae: Dynastini). Bulletin of the University of Nebraska State Museum, 16, $1-506$.

Ratcliffe, B. C., Cave, R D. y Paucar-Cabrera, A. 2006. The dynastine scarab beetles of Ecuador (Coleoptera: Scarabaeidae: Dynastinae). Bulletin of the University of Nebraska State Museum 32: 1-586.

Restrepo, H., Morón, M. A., Vallejo, F., Pardo-Locarno, L. C. y López-Ávila, A., 2003. Catálogo De Coleóptera Melolonthidae (Scarabaeidae Pleurosticti) de Colombia. Folia Entomológica Mexicana, 42(2): 239-263.

Ritcher, O. P. 1940. Kentuky White Grubs. Kentuky Agricultural Station. Bulletin 401: 71-157.

Ritcher, O. P. 1944. Dynastinae of North America With Descriptions of the larvae and Keys to Genera and Species (Coleoptera: Scarabaeidae). Kentuky Agricultural Station. Bulletin 467: 1-56.

Ritcher, O. P. 1966. White Grubs and their Allies. Oregon State University Press: 219 pp.

Snodgrass, RE. 1967. Insects their Ways and Means of Living. Dover Publications, Inc. New York. 362 p. 